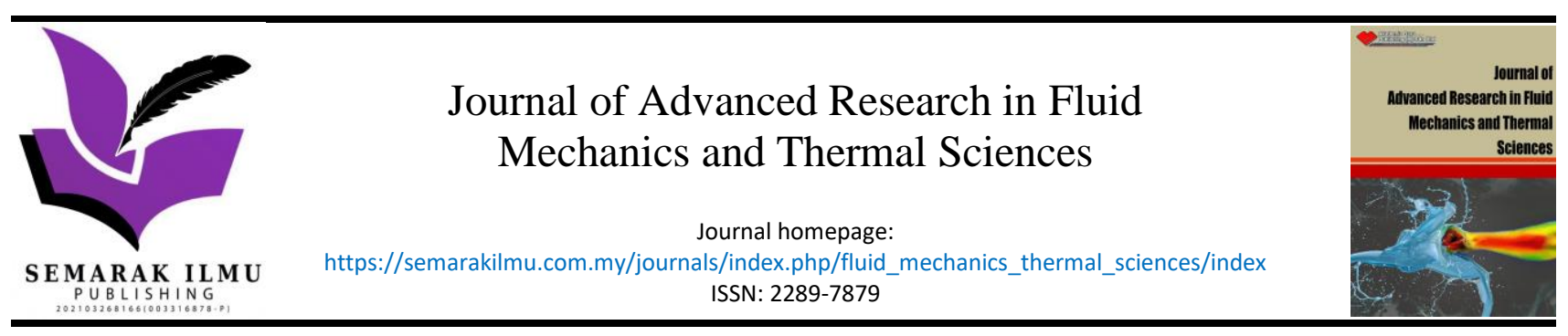

\title{
A Review on Heat Transfer Enhancement of Solar Air Heater Using Various Artificial Roughed Geometries
}

\author{
Sudharani Panda ${ }^{1}$, Rakesh Kumar ${ }^{1, *}$ \\ 1 Department of Mechanical Engineering, IIT (ISM) Dhanbad, Dhanbad, Jharkhand-826004, India
}

ARTICLE INFO ABSTRACT

\section{Article history:}

Received 27 June 2021

Received in revised form 5 October 2021

Accepted 9 October 2021

Available online 20 November 2021

\section{Keywords:}

Heat transfer rate; friction factor; ribs; solar air heater; artificial roughness

\begin{abstract}
Solar air heater acts as one of the important components in utilization of solar energy. The air heater absorbs the irradiance and converts it into heat energy at the absorbing surface. The thermal energy is further use in heating flowing air through the duct. Solar air heaters are cost effective as well as simple in design. Solar air heater can be used in space heating, timber seasoning and agricultural drying. In spite of all these advantages the solar air heater has certain challenges such as the air has low heat transfer coefficient. The heat transfer rate from the heated absorber surface to the air is low. Hence in order to enhance the heat transfer coefficient the surface area either increases or the flow made to be turbulent. In order to do so the artificial roughened element must be incorporated on the heated surface. The use of artificial roughness is considered as an effective technique to enhance the heat transfer rate of fluid flowing through the duct of solar air heater. The heat transfer and friction characteristics of number of roughness geometries incorporated solar air heater have been investigated. In this paper an attempt has been made to review on element geometries used as artificial roughness in solar air heater in order to improve thermal and thermo hydraulic performance of solar air heater ducts.
\end{abstract}

\section{Introduction}

Energy in various forms plays an important role in world wide economic progress and industrialization [1, 2]. Most of the energy demands full fill by conventional source such as coal, oil and natural gas. Although most of the energy demand across the globe can be full filled by the use of fossils fuel, some challenges have also associated with the use of it such as: (a) the resources of fossils fuel are limited as it is comes under non renwable source of energy [3, 4], (b) the fossils fuels owened by few countries only [3], (c) price instability of fossils fuel [3], (d) it causes environmetal issues like global warming [3,4] and (e) global energy security [3,5]. To overcome this energy crisis the renewable source like sun, tide, wind, alternative fuels (i.e. Biodiesel) must be utilized [6]. Among all the Renewable source of energy solar energy is considered to be vital energy source to meet the increased energy demand. Solar energy is the radiant light and heat which comes from the sun and

\footnotetext{
* Corresponding author.

E-mail address: rakesh@iitism.ac.in
}

https://doi.org/10.37934/arfmts.89.1.92133 
reaches to the earth surface. About half of the solar radiation reaches to the earth surface passing through the space. So, it is essential to capture the solar energy and utilized it effectively. Therefore, different technologies by the help of which the solar energy can be harnessed such as (a) passive energy, (b) active energy, (c) photovoltaic energy, (d) solar thermal energy and (e) concentrated solar power.

The solar energy which is harnessed by different technologies have several advantages such as, (a) it is freely available, (b) it is infinitely available, (c) it doesn't emit any pollutant to the atmosphere, (d) it does not contribute to greenhouse effect, acid rain or smog, (e) it is cheaper as compared to electricity, (g) it does not require any transportation and (h) it can able to overcome fossil depletion problem. Due to these benefits of the solar energy, it has been used for various applications such as, heating of water, heating of building, drying of agricultural product, space heating, thermal power plant and many more.

The solar energy which is used for many purposes can be harnessed by several thermal technologies. Among several solar thermal technologies, solar air heating system is one of the widely used solar thermal technologies due to its design simplicity and cost effectiveness. The air leakage, corrosion and freezing are not represented as a major problem in solar air heater [7]. There is no boiling and pressure issue in solar air heater [7]. They do not require high technology for their maintenance and repair [7]. Solar air heating system collects the solar radiation then converts it into heat in the absorbing surface and transfer the heat to the air flowing through the system. Solar air heating system is used for drying of agricultural product, timber seasoning, space heating and thermal power plant. etc. A typical soar air heating system is schematically shown in Figure 1. It consists of seven components, inlet duct, outlet duct, absorber tray, glazing, rigid frame, insulation and wooden cabinet.

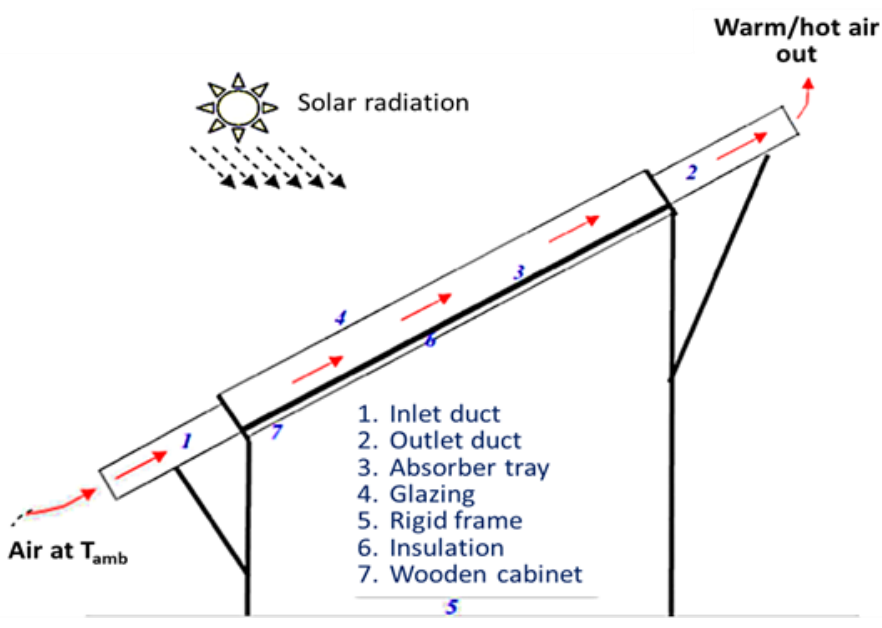

Fig. 1. Major components of a solar air heating system [7]

A layout of glazing is supplied on the top surface of the glass to absorb more solar irradiation. The material generally used as glazing are acrylic, tempered glass, polycarbonate etc. [8]. The solar radiation first incident over the glazing from the glazing it will be transferred to the solar absorber plate. The solar absorber plate which absorbs the radiations must be made of highly thermal conducting material, must be of unique combination of light weight, high strength and ease of fabrication (i.e. aluminum alloy) [9]. In solar air heating system, this absorber plate is an important component [10]. It decides the effectiveness of heat transfer to the air to be heated. This effectiveness is depending on the design and nature of the absorber plate. Therefore, the solar 
absorber plate is again classified into two types one is porous and other is non-porous type. Figure 2 represents the types of solar absorber plate. Porous type has porous absorber which may include slit and expanded metal, overlapped glass plate absorber as shown in Figure 2(a). In non-porous type, air does not pass through below the absorber plate but air may flow above the plate as shown in Figure 2(b).

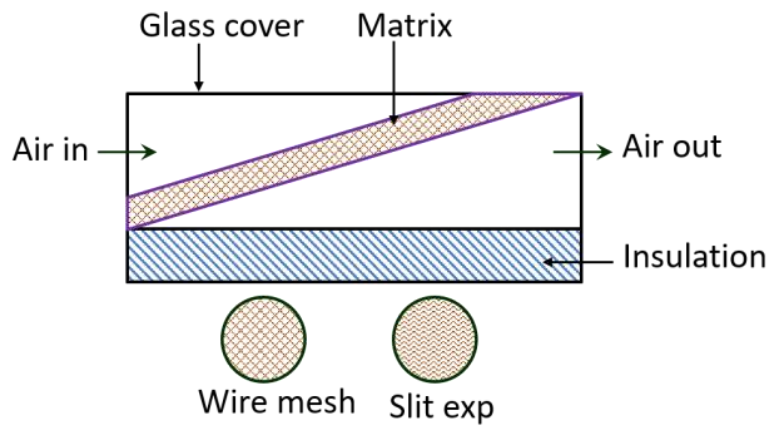

(a)

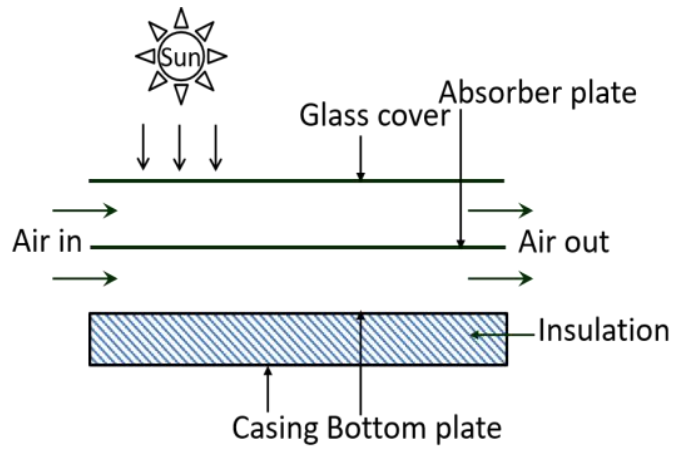

(b)

Fig. 2. Absorber plate; (a) porous $[11,12]$ and (b) non-porous

The air at ambient condition enters to the solar air heating system through inlet duct and the hot air comes out from the solar air heating system through outlet duct. The insulations are provided in the side and bottom walls of the ducts to minimize the heat loss to the surrounding [8]. The whole arrangement is placed in a wooden cabinet and the wooden cabinet is mounted over the rigid frame as illustrated in Figure 1.

\subsection{Enhancement of Heat Transfer}

The heat transfer rate of solar air heating system needs to be enhanced due to following reasons

i. To increase the heat transfer coefficient between the heated absorber plate and flowing air as the heat transfer coefficient of the air is very low.

ii. To increase thermo hydraulic performance.

iii. To reduce friction factor.

iv. To utilize maximum amount energy absorbed by the solar air heater.

v. To increase life of the component of solar air heating system.

vi. To maintain compactness of the solar air heating system.

To fulfill all these objectives the solar air heating system must be incorporated with some artificial roughness element such as: (a) Ribturbulator. (b) Finned/Baffles. (c) Dimple (d) Protrusion.

In the solar air heater the presence of laminar sub layer between the absorber plate and flowing air is considered as main cause of thermal resistance [13]. The artificial roughness element helps to disturb the flow in laminar sub layer and to reduce the thermal resistance [13]. Due to which the heat transfer rate increased or by the addition of these elements the convection heat transfer co-efficient of air increases [14].

The review is focused on experimental and numerical investigations that have been reported in the literature by various researchers on artificial roughness in solar air heaters. Further, many investigators have attempted to design a roughness element that helps in the enhancement of convective heat transfer with minimum friction penalty in solar air heaters. In the present study, the investigation has been made to categorize and carry out a comprehensive review of reported roughness geometries used to create artificial roughness. The effect of artificial roughness 
geometries in the solar air heater absorber plate on thermal performance has been analyzed as per the availability in the literature. Most of the developed artificial geometries and their configuration that influence the normalized Nusselt number have also been addressed. Besides, the numerical and experimental investigations on different structures of various roughness geometries used in solar absorber plates relative to flat ones have been discussed. Finally, few future scopes have been highlighted in the present investigation.

\section{Mathematical Model of Conventional Solar Air Heater}

The solar air heater can be designed efficiently by analyzing the thermal and hydraulic performance of solar air heater. The thermal performance related with heat transfer rate of solar collector whereas the hydraulic performance related to pressure penalty in a duct.

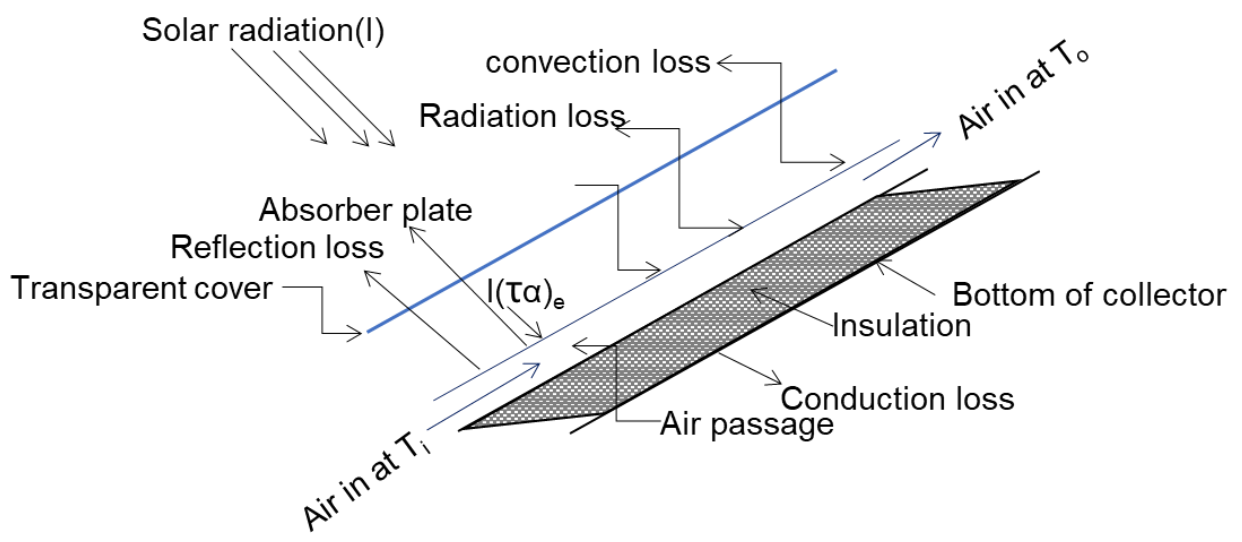

Fig. 3. Conventional solar air heater [13]

\subsection{Thermal Performance}

To evaluate the thermal performance of a solar air heater Hottel-Whiller-Bliss equation reported by Duffie and Beckman [13] is commonly used.

$\mathrm{Q}_{\mathrm{u}}=\mathrm{A}_{c} \mathrm{~F}_{\mathrm{R}}\left[\mathrm{I}(\tau \alpha)_{\mathrm{e}}-\mathrm{U}_{\mathrm{L}}\left(\mathrm{T}_{\mathrm{i}}-\mathrm{T}_{\mathrm{a}}\right)\right]$

Or $q_{u}=Q_{u} / A_{c}=F_{R}\left[I(\tau \alpha)_{e}-U_{L}\left(T_{i}-T_{a}\right)\right]$

The rate of useful energy gain by the flowing air through duct of a solar air heater can also be calculated by using the following equation

$Q_{u}=m^{0} c_{p}\left(T_{0}-T_{i}\right)=h A_{c}\left(T_{p m}-T_{a m}\right)$

As per the discussion above, heat transfer coefficient ( $h$ ) can be increased by applying artificial roughness on the surface of absorber plate. It can be represented in non-dimensional form by using the following relationship of Nusselt number $(\mathrm{Nu})$ reported by Duffie and Beckman[13].

$\mathrm{Nu}=\mathrm{hl} / \mathrm{k}$

Thermal efficiency of solar air heater can be expressed by the following equations 
$\eta_{\text {th }}=q_{u} / I=F_{R}\left[I(\tau \alpha)_{e}-U_{L}\left(T i-T_{a} / I\right)\right]$

\subsection{Hydraulic Performance}

The hydraulic performance always calculated by considering the pressure drop $(\Delta p)$ in the duct. With the increase in pressure drop the power consumption by fan or blower increases to propel the air through the duct. Pressure drop can be represented in non-dimensional form by using the following relation of friction factor ( $f$ ), reported by Frank and Mark [13].

$f=(\Delta p) D_{h} / 2 p l v^{2}$

\subsection{Thermo Hydraulic Performance}

It is essential to design the collector in such a way that it should transfer maximum heat energy to the flowing fluid with minimum power consumption by fan or blower. So in order to analyze overall performance of solar air heater thermo hydraulic performance must be evaluated by considering both thermal and hydraulic characteristics of the collector simultaneously. The thermo hydraulic performance of solar air heater can be evaluated using the formula given by Webb, Eckert and Goldstein [15].

$\eta t_{h p}=\left(N u / N u_{s}\right) /\left(f / f_{s}\right)^{1 / 3}$

\section{Methodology on Artificial Roughness}

In order to achieve higher heat transfer coefficients it is essential to create turbulent flow at the heat transferring surface. However in order to create turbulence the energy required is supplied by fan or blower and after consuming excess power the air has to flow through the duct. Therefore the turbulence must be created only in the region adjacent to the heat transferring surface or in the laminar sub layers region where the heat transfer takes place. The flow should not be unduly disturbed so as to avoid excessive friction losses. This can be done by maintain the height of the roughness element small as compared to the duct dimension. There are several parameters that characterize the arrangement and shape of the roughness element. Among many parameters the roughness height $(e)$ and roughness pitch $(p)$ are two most important parameters. These parameters are specified in non dimensional form as relative roughness height (e/D) and relative roughness pitch $(p / e)$ respectively. The other parameters are Reynolds number, cross section of rib, attack angle, chamfering and combined turbulence promoters.

\section{Roughness Geometries Used in Solar Air Heater}

The solar air heaters which are incorporated with artificial roughness in the form of fixing small diameter wires, machining ribs of different shapes, forming dimple/protrusion have been investigated to evaluate the heat transfer enhancement rate of absorber plate. Many literatures on application of artificial roughness in a solar air heater include wide range of roughness geometries to study heat transfer and friction characteristics. The various roughness geometries general arrangement reported by several investigators can be classified into five types: (i) Wire fixation, (ii) Rib formation by machining process, (iii) Wire mesh or expanded metal mesh fixation, (iv) 
Dimple/Protrusion formation, (v) Fins and Baffles. These types of roughness geometries are listed in Table 1.

\section{Table 1}

Various roughness geometries incorporated with solar air heater

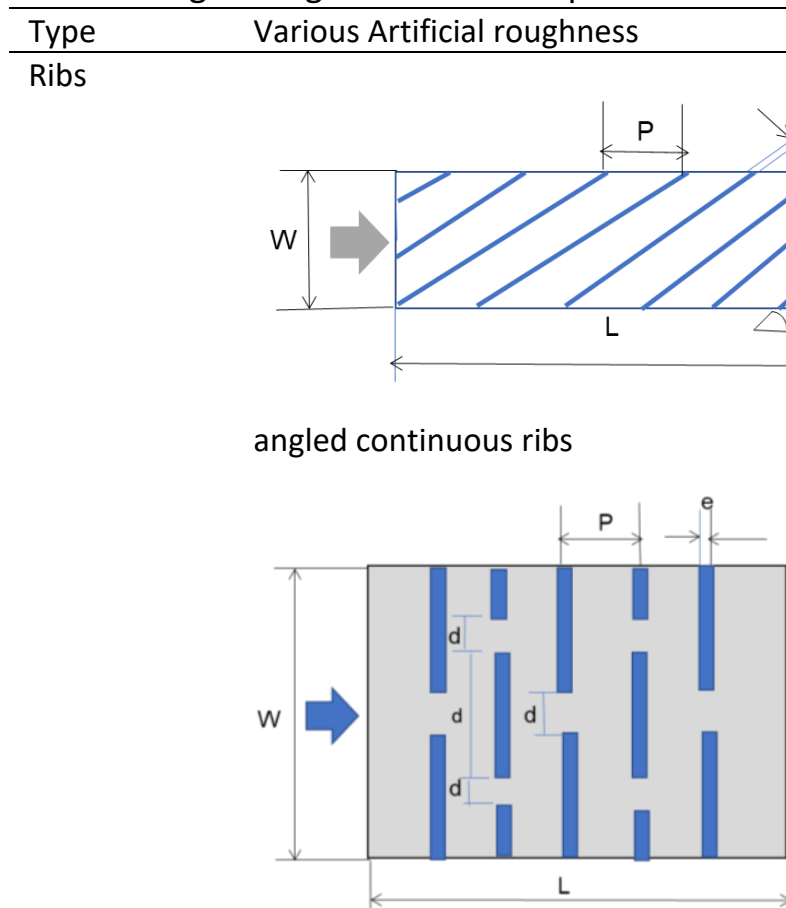

transverse broken ribs

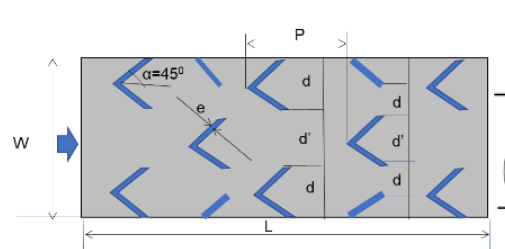

V-shaped rib

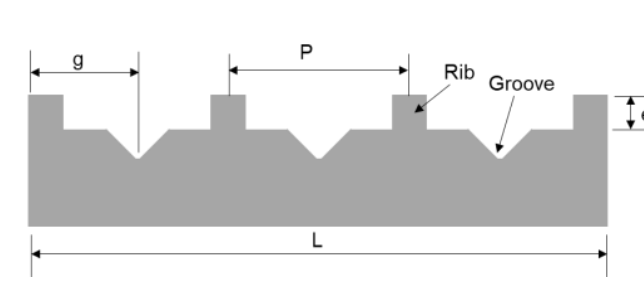

rib groove arrangement

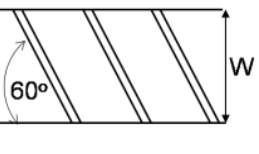

inclined non-continuous rib transverse continuous ribs

Ref.

[16]

[16]

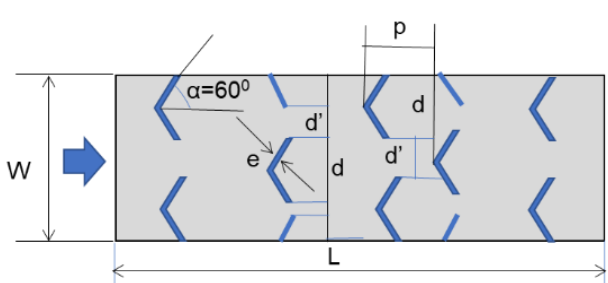

discrete-shaped rib

$[13,16]$

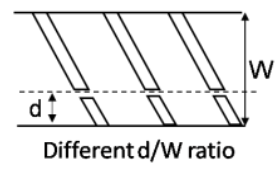

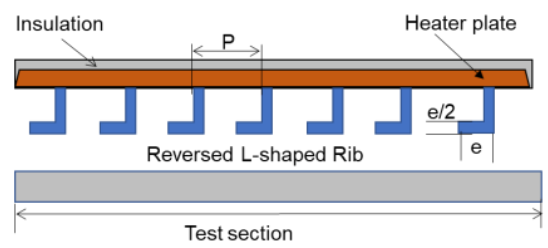

with L-Shaped rib

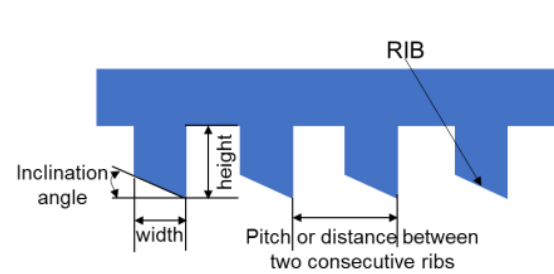

chamfered rib solar absorber plate
Half angle of $\quad V$ shaped rib

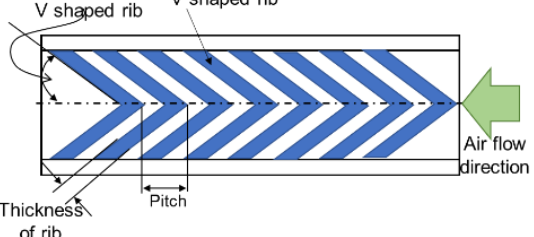

of rib

60 degree-shaped rib 


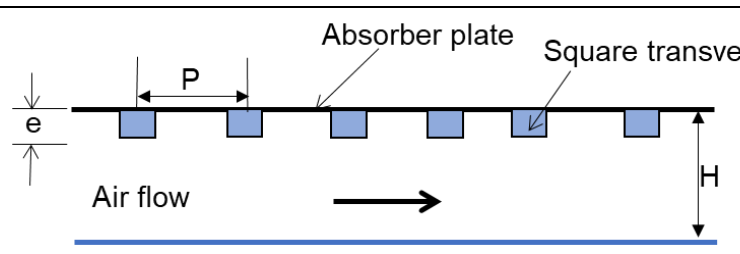

Square transverse ribs solar absorber plate

Mesh

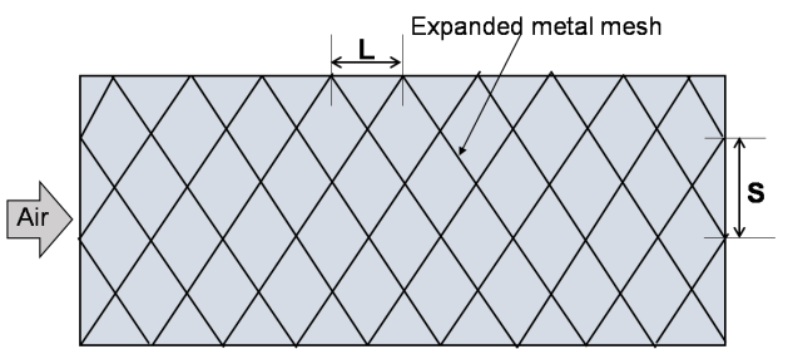

Expanded Metal mesh

Dimple

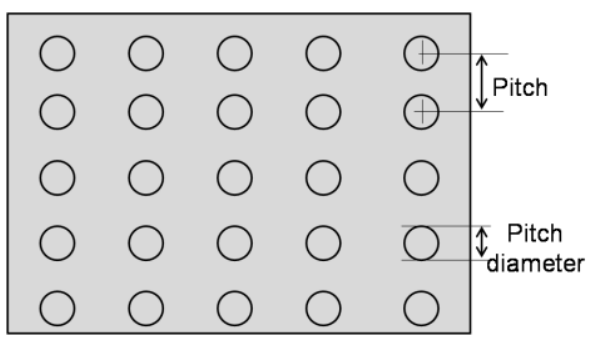

Absorber plate with Dimple-shaped geometry

W shape rib

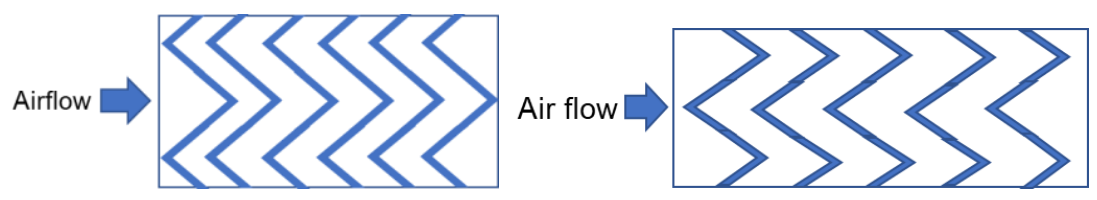

Vshape groove

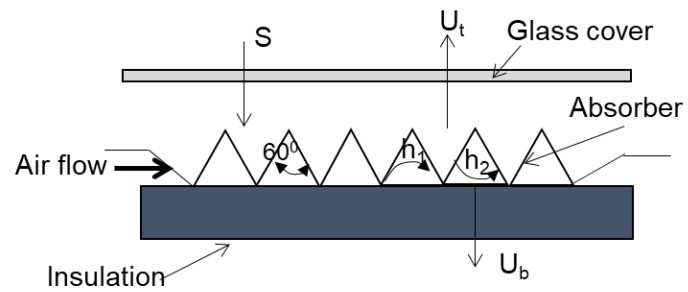

Corrugated

collector

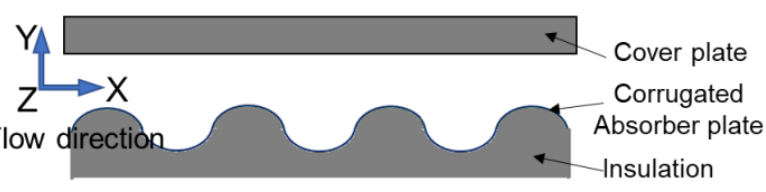

Finned collector

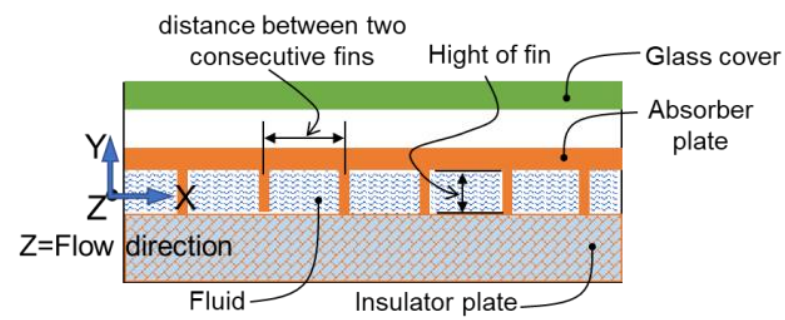




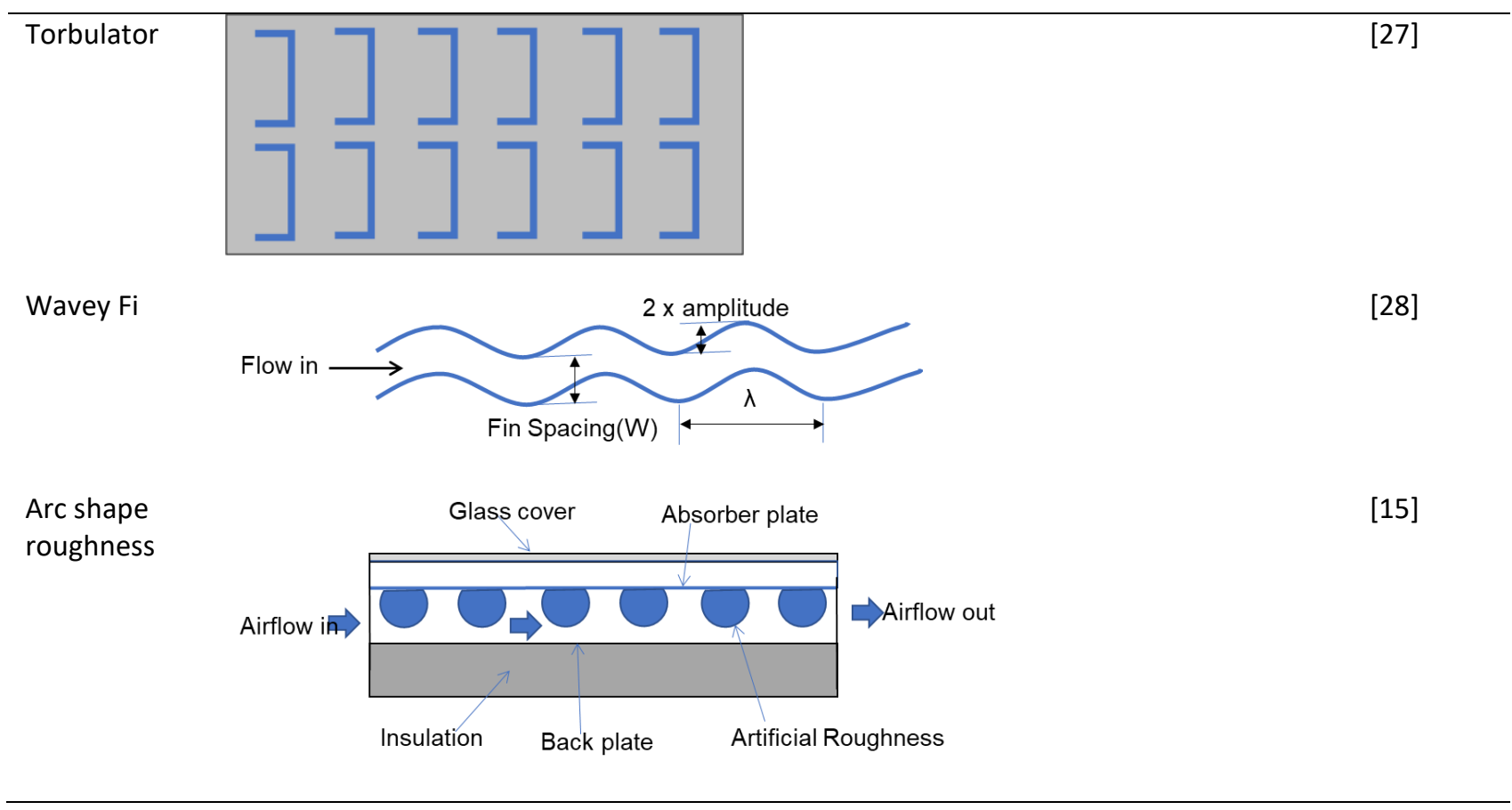

\subsection{Wire Fixation}

The heat transfer enhancement and friction loss characteristics of solar absorber plate have been studied by various investigators by fixing protruding wires of different shape, size and orientation as an artificial roughness element on absorber plate.

\subsubsection{Transverse continuous and broken ribs}

Alam et al., [29] investigated numerically the effect of relative ribs pitch, relative ribs height of solar air heater which is protruded with conical protrusion ribs on Nusselt number and friction factor. The relative ribs height $(\mathrm{p} / \mathrm{e})$ has been varied from 6 to 12 and the relative ribs height (e/D) varied from 0.020 to 0.044 respectively. The investigation has been carried out by varying the Reynolds number from 4000 to 16000 . The numerical simulation has been carried out using CFD code ANSYS FLUENT 16.0.The boundary condition has been applied to the computational domain are: (i) $A$ uniform heat flux of $1000 \mathrm{~W} / \mathrm{m}^{2}$ has been supplied to the upper surface of the absorber plate. (ii) The bottom and side wall of the absorber plate has been considered smooth and insulated. (iii) The no slip boundary condition has been employed on the smooth wall. (iv) At the inlet the inlet velocity and at out let pressure outlet boundary condition has been applied. The coupling of velocity and pressure has been carried out using SIMPLE algorithm. The second order upwind discretization technique has been adopted. The RNG $k-\epsilon$ model has been applied to capture the intensity of turbulent flow structure. The Nusselt number of solar air heater equipped with conical and spherical ribs have higher value than the smooth duct obtained using Gnielinski correlation equation and Dittus-Boelter correlation as function of Reynolds number for relative ribs height of 0.0289 and relative ribs pitch of 10 . This is due to higher turbulence level in the flow due to the presence of conical and spherical protrusion on solar air heater. The Nusselt number of conical protrusion solar air heater is higher as compared to spherical protrusion solar air heater. The conical shape ribs introduce high turbulence in the flow as well as it provides higher reattachment point on the surface. The strong reattachment on surfaces disturbs the viscous sub layer. Due to higher turbulence level it promotes mixing of flow near surface to core flow. The reattachment points present in spherical roughened rib are too weak 
so the heat transfer rate is low. With the increase in Reynolds number value the thickness of laminar sub layer decreases hence the friction factor decreases for both smooth duct as well as roughened duct. The presence of sharp edges of conical rib induces higher pressure drop in the flow. The Nusselt number of conical rib is 1.3 times than that of spherical rib roughened surface and the friction factor of conical rib roughened surface is 1.07 times higher. The Nusselt number of rib roughened surface increases with the increase in Reynolds number due to increase in intensity of turbulent kinetic energy. The higher intensity of turbulent kinetic energy has been obtained at downstream of all the ribs. The higher turbulent kinetic energy region has been pronounced in ribs due to large velocity gradient in flow. The maximum turbulent kinetic energy value lies in between $0.26 \mathrm{j} / \mathrm{kg}$ to $1.74 \mathrm{kj} / \mathrm{kg}$ when the Reynolds number varies from 4000 to 16000 . For a fixed value of relative rib pitch $(p / e=10)$ and at a relative ribs height of 0.044 the Nusselt number value as well as friction factor increases with the increase in Reynolds number. The Nusselt number and friction factor value at relative rib height is 0.020 is minimum. The turbulence level increases with the increase in relative rib height due to local velocity profile which enhance the mixing rate of flow between the ribs. Due to increase in relative roughness height more disruption to flow occurs. This causes more dynamics pressure loss of fluid. Hence the friction factor increases with the increase of relative roughness height from 0.020 to 0.044 . At a fixed value of relative ribs height of 0.0289 and with the increase in relative ribs pitch(6 to 10) for all value of Reynolds number the Nusselt number increases. When the relative rib pitch is 6 the Nusselt number is minimum. For relative rib pitch 10 the Nusselt number value is maximum. When the relative rib pitch value is beyond 10 the Nusselt number value decreases at all value of Reynolds number. At relative pitch value of 10 the Nusselt number is maximum due to lateral movement of air. The lateral movement of air reduces the recirculation zone with the decrease in recirculation zone better heat transfer area can be achieved. The friction factor decreases with the increase in relative pitch ratio as the resistance to flow decreases. The minimum and maximum value of friction factor has been obtained at relative pitch of 12 and 6 respectively. The thermal efficiency increases with the increase in Reynolds number for all value of relative rib height. The efficiency of roughened solar air heater is higher as compared to the smooth plate solar air heater. The thermal efficiency is maximum at relative roughness height of 0.044 and minimum at 0.020 for all Reynolds number range. The maximum value of thermal efficiency is obtained at relative rib pitch 10 and minimum at 6.

Sahu et al., [30] studied experimentally the heat transfer co-efficient of solar air heater which is incorporated with 90 degree broken transverse ribs on the absorber plate. The roughened wall has been supplied with heat whereas the remaining three walls have been maintained at insulated condition. The roughened wall roughness with pitch has been varied from $10-30 \mathrm{~mm}$. The height of the rib has been maintained at $1.5 \mathrm{~mm}$. The duct aspect ratio has been maintained at 8 . The air flow rate correspond to Reynolds number has been varied from 3000-12,000. The Nusselt number value for the roughness with pitch value of 10,20 and $30 \mathrm{~mm}$ increases with the increase in Reynolds number. The Nusselt number value has been found to be maximum at roughness with pitch value of $20 \mathrm{~mm}$. The Nusselt number value is the ratio of conduction resistance to convective resistance. With the increase in Reynolds number the thickness in boundary layer decreases. The reduction in boundary layer thickness decreases the convective resistance which helps to increase the Nusselt number value. At lower Reynolds number the enhancement rate of Nusselt number over the smooth duct wall is small. At lower Reynolds number value $(\mathrm{Re}<5000)$ the Nusselt number value of smooth duct is equal to the rough duct. It is due to the increase in laminar sub layer thickness due to retardation of flow by the roughness element. The heat transfer coefficient value increases with the increase in Reynolds number value for a constant pitch value. At low Reynolds number value $(\operatorname{Re}<$ 5000) the increment rate of heat transfer coefficient value is negligible as the boundary layer remains 
unbreakable. Due to this thermal resistance to heat flow increases and heat transfer coefficient value decreases. The Nusselt number value increases rapidly with the increase in value of pitch from 10 to $20 \mathrm{~mm}$. The convection heat transfer coefficient value increases rapidly with the increase in value of pitch from 10 to $20 \mathrm{~mm}$. At low value of pitch the reattachment point which is the main reason for heat transfer enhancement forms properly on the surface of the absorber plate. Whereas with the increase in pitch value (more than $20 \mathrm{~mm}$ ) the reattachment decreases as a results of which the heat transfer rate decreases. At very low pitch value the reattachment point cannot be formed hence the heat transfer rate may be decreased. The highest thermal efficiency value of $83.5 \%$ has been obtained at pitch value of $20 \mathrm{~mm}$. The 90 degree broken ribs incorporated solar air heater heat transfer coefficient is 1.25-1.4 times as compared to the smooth rectangular duct under similar operating condition at higher Reynolds number.

Tanda [16] studied experimentally the heat transfer characteristics and friction factors for a rectangular duct whose one wall has been incorporated with repeated ribs. The wall incorporated with repeated ribs has been supplied with constant heat flux and the remaining three walls have been provided with insulation. The four different types ribs named as angled continuous ribs, transverse continuous, broken ribs and discrete $\mathrm{v}$-shaped ribs have been incorporated one wall of the channel. The heat transfer rate for all the rib incorporated solar air heater increases at the same Reynolds ranges. The broken and continuous ribs heat transfer enhancement rate is higher among the rest rib roughened solar air heater. The friction factor for both broken and transverse continuous ribs is highest. The friction factor increase rate is higher at relatively low Reynolds number value for the roughened channels and the friction factor value is higher at relatively higher Reynolds number value for the smooth channel. When the solar radiation intensity is higher at that time the maximum efficiency is obtained by roughened solar air heater as compared to the smooth channels at Reynolds number range $15,000-16,000$. With the decrease in solar radiation intensity the effective efficiency decreases for all the rib configurations. With the decrease in solar radiation intensity the heat transfer to the air reduced due to decrease in heat absorption by solar collector or due to decrease in temperature of the absorber plate. The decrease in heat transfer to the air becomes more prominent with the increase in Reynolds number value when the intensity of solar radiation is low. The ratio of Nusselt number of solar air heater with rib and without rib is denoted by the letter $N_{1}$. When the pumping power is same and only the Reynolds number varies the $\mathrm{N}_{1}$ value for all the configuration is more than one but the degree in increase in $\mathrm{N}_{1}$ value with the increase in Reynolds number value is reduced. The transverse broken ribs produced highest $\mathrm{N}_{1}$ value among all the ribs arrangements at a constant supply of pumping power. At relatively low Reynolds number value a relative maximum value of effective efficiency has been obtained for ribs roughened solar air heater and for smooth duct solar air heater at high Reynolds number. The ratio of effective efficiency of rib roughened solar air heater to smooth duct solar air heater has been denoted by letter $\mathrm{N}_{2}$ augmented significantly for rib roughened solar air heater at lowest Reynolds number value and with the increase in Reynolds number vanishes as the Reynolds number value is sensitive to solar radiation intensity after a certain range.

Gawande et al., [18] investigated the thermal performance of solar air heater experimentally as well as numerically whose absorber plate is incorporated with reverse L-shaped ribs. The design variables have been considered for evaluating thermal performance named as relative roughness pitch, Reynolds number, heat flux and relative roughness height. The design variables such as relative roughness pitch and Reynolds number has been varied from 7.14 to 17.86 and from 3800 to 18,000 respectively. A heat flux of $1000 \mathrm{w} / \mathrm{m}^{2}$ has been supplied to the absorber plate. A constant value of relative roughness height 0.042 has been maintained throughout the investigation. To evaluate the effect of relative roughness pitch, Reynolds number on heat transfer and friction factor 
characteristics in L-shaped rib incorporated solar absorber plate a two dimensional CFD simulation has been carried out. The two dimensional CFD simulation has been carried out using CFD code ANSYS FLUENT of version 14.1.The RNG $k-\epsilon$ turbulence model has been used to solve the turbulence terms in the governing equations. For different value of relative roughness pitch and at a constant value of relative roughness height the heat transfer rate in a reversed L-shaped rib incorporated absorber plate solar air heater increases with the increase in Reynolds number. The rate of heat transfer is higher as compared to the smooth plate solar air heater. The averaged Nusselt number value of Lshaped rib incorporated solar air heater increases with the increase in Reynolds number due to following reason: (i) when the Reynolds number increases the thickness of laminar sub layer decreases (decrease in laminar sub layer means less resistance to heat flow) (ii) The velocity increases with the increase in Reynolds number. (iii) The turbulence is produced with the increase in velocity. (iv) This turbulence helps to creates vortices on the top surface of the reversed L-shaped rib incorporated absorber plate which helps to transfer heat from the hot surface of the absorber plate to the cold fluid available in the center. (v) The reversed L-shaped rib disturbs the boundary layer developed due to which the turbulent kinetic energy and turbulence intensity increases. The average Nusselt number value increases for the relative roughness pitch range 7.14 to 17.86 but the maximum value of average Nusselt number value is obtained for the relative roughness pitch value of 7.14 at a fixed value of relative roughness height. With the decrease in relative roughness pitch the reattachment point increases due to the presence of more number of ribs spacing on the absorber plate. The average turbulent intensity as well as the flow acceleration increases with the decrease in relative roughness pitch. Whereas with the increase in relative roughness pitch the number ribs on the absorber plate decreases due to which reattachment point decreases and Averaged Nusselt number decreases. The maximum enhancement of Nusselt number for a fixed value of relative roughness height 0.042 has been obtained at relative roughness pitch 7.14 and Reynolds number 15,000. The maximum enhancement is about 2.827 times over the smooth duct. The addition of reversed L-shaped ribs on absorber plate of solar air heater provides obstruction to the flow. The obstruction to the flow introduces the phenomena such as flow separation and reattachment which is responsible for large pressure drop. The increase in pressure drop increases the friction factor in roughened solar air heater as compared to the smooth duct. The friction factor decreases with the increase in Reynolds number value due to following reasons: (i) The laminar sub layer thickness or the viscous sub layer thickness decreases with the increase in Reynolds number value. (ii) With the increase in Reynolds number value the turbulent intensity increases which enhance the heat transfer rate with less value of friction factor. The maximum enhancement of friction factor has been reported at relative roughness pitch 7.14, relative roughness height 0.042 and at Reynolds number 3800. The maximum enhancement is about 3.424 than the smooth duct. The turbulent kinetic energy and turbulent intensity reaches to a maximum value near the absorber plate and in between the region of first and second ribs. The turbulent kinetic energy and turbulence intensity decreases with the increase in distance from the absorber plate. At the inlet the flow is developing hence the shear is stronger in the boundary layer. This causes higher turbulence in the tip of the first L-shaped ribs. The flow from developing regions enters to the developed regions after crossing the entrance length. When the flow becomes fully developed the shear in the boundary layer decreases and moving away from the absorber plate. Hence intensity of turbulence decreases. The heat transfer enhancement increases with the increase in turbulent intensity as intensity of shear layer is greater in the neighborhood of the ribs as compared to the smooth solar air heater. The heat transfer enhancement in L-shaped rib incorporated solar air heater is due to movement of hot fluid from the hot surface of the absorber plate and clod fluid momentum towards the heated surface of absorber plate. The optimum value of thermo hydraulic performance parameter (when the power 
requirement is minimum) by choosing the best configuration of reversed L-shaped ribs lies between 1.62 and 1.90 in the given range of design variable. The optimum value of thermo hydraulic performance parameter is $\mathbf{1 . 9 0}$ for L-shaped rib incorporated solar air heater when the relative roughness pitch is 7.14 , relative roughness height is 0.042 and Reynolds number is 15,000 . The simulation result is in good agreement with the experimental result.

\subsubsection{Inclined and V-shaped or staggered ribs}

Kumar et al., [31] carried out a comprehensive literature review on the effect of various artificial roughness elements used in the form of repeated rib on heat transfer and friction factor characteristics of solar air heater. It has been concluded that the artificial roughness element attached to the solar air heater duct has been categorized into various types depending on the shape, size, orientation and arrangement. The general types of artificial roughness element which are used by many investigators are fixing wires, rib formation by machining process, expanded metal mesh ribs and creating dimple shape geometries. The heat transfer coefficient of transverse rib is enhanced due to the phenomenon such as flow separation, formation of vortices in the upstream and downstream rib and reattachment of flow in the inter rib spaces. The inclined rib enhance the heat transfer rate due to the movement of vortices along the rib and formation of secondary flow cell which results high heat flow zone near the leading end. The $\mathrm{V}$-shaped of a long angled rib produce two secondary flow cells which further enhance the heat flow as compared to the inclined rib. The continuous inclined rib with gap enhance the heat transfer rate by breaking the secondary flow and producing higher level of turbulence in the fluid downstream of the rib. A gap in the limb of v-shape rib further enhances the heat transfer rate by breaking the secondary flow and producing high level of turbulence in the fluid downstream of the rib. The presence of multiple v-shape rib along the width of the plate enhanced the heat transfer rate by producing more number of secondary flows. The multiple v-shaped ribs have higher thermo hydraulic performance as compared to other roughness element.

Ghritlahre et al., [32] investigated experimentally the effect of arc shaped roughened solar air heater on thermal performance and heat transfer rate. The studied has been carried out for apex up and apex down oriented arc shaped wire rib solar air heater. The arc shaped roughness with relative roughness pitch, relative roughness height, rib roughness and arc angle 10, 0.0395, 2.5 and 60 degree respectively has been taken for experimentation. The thermal performance and heat transfer rate has been evaluated by varying the mass flow rate from 0.007 to $0.022 \mathrm{~kg} / \mathrm{s}$. It has been observed that the solar intensity increases up to noon and starts falling on wards. The maximum solar intensity has been obtained for apex up arc shaped solar air heater. The maximum solar intensity of $853.3 \mathrm{w} / \mathrm{m}^{2}$ has been obtained for apex up arc shaped solar air heater at 12.30h.The maximum solar intensity of $756.1 \mathrm{~W} / \mathrm{m}^{2}$ has been achieved by apex down arc shaped solar air heater at $12.30 \mathrm{~h}$. It has been concluded from the investigation that the outlet temperature for both types of solar air heater increases with time and it starts decreasing in the afternoon. The maximum outlet temperature has been obtained at lower mass flow rate for apex up arc shaped solar air heater in between 11.30 to $13,00 \mathrm{~h}$ as the solar intensity is maximum during this period. The maximum outlet temperature has been obtained at lower mass flow rate as the residence time of cold fluid over the heat transferring surface is higher hence more amount of heat will be absorbed by the flowing fluid. The maximum temperature difference has been achieved at $12.00-13.00 \mathrm{~h}$ as a result of which maximum thermal efficiency has been obtained during this period. The maximum thermal efficiency has been obtained as $43.5 \%, 41 \%$ and $25.2 \%$ for upstream, downstream and smooth solar air heater at $12.00-13.00 \mathrm{~h}$. The apex upstream solar air heater is $72.2 \%$ and apex down solar air heater is $63.1 \%$ more efficient as 
compared to smooth solar air heater. The heat transfer rate of apex up solar air heater is higher as compared to the apex down solar air heater.

Aharwal et al., [21] investigated heat transfer and friction characteristics of solar air heater which has been incorporated with integral repeated discrete square ribs. The duct which has been taken for investigation maintain a width to height ratio of 5.83. The effect of geometrical parameter on performance of solar air heater such as gap width, gap position has been investigated. The relative gap position $(\mathrm{d} / \mathrm{w})$ and relative gap width $(\mathrm{g} / \mathrm{e})$ has been varied from 0.16 to 0.5 and 0.5 to 2.0 respectively for analyzing its effect on performance of solar air heater. The Reynolds number has been varied from 3000 to 18,000 , whereas the relative roughness pitch (p/e) has been varied from 4-10 and relative roughness height (e/D) has been varied from 0.018-0.037 respectively. The angle of attack has been varied from $30^{\circ}-90^{\circ}$. From the investigation it has been concluded that the Nusselt number value of continuous rib with gap is always higher as compared to the continuous rib without gap. The Nusselt number of continuous rib with gap has been increases for the variation of relative gap position from 0.16 to 0.25 . The Nusselt number decreases with the increase in relative gap position further. The enhancement of Nusselt number is 1.53 times to 2.83 times than that of smooth duct. When the inclined rib in a rectangular duct is introduced the secondary flow along the rib length is formed. It allows the working fluid to travel from the leading edge to trailing edge of the rib. The working fluid which is passed over the inclined rib than heated and the thickness of the boundary layer increases. The main flow which is passing through the gap is fully developed flow with thicker boundary layer consisting of viscous sub layer. Due to the presence of gap the secondary flow joins with the main flow to accelerate it which energized the retarded boundary layer flow along the surface. This increases the heat transfer through the gap width area behind the rib. The Nusselt number increases up to the increase of relative gap width up to 1.0 beyond this value it starts decreasing. The minimum value of Nusselt number is obtained at relative width gap of 2.0. The introduction of inclined rib and gap are two primary reason of heat transfer enhancement in inclined rib incorporated solar air heater. The addition of rib induces secondary flow whereas the gap promotes the local turbulence and mixing along the gap flow region. If the gap will be too small the sufficient flow of secondary flow cannot be possible hence the turbulence level will remain low. The maximum Nusselt number value is obtained for a relative gap position of 0.25 and relative gap width of 1.0 at relative pitch of 8.0. It is about 2.83 times that of the smooth surface. The maximum Nusselt number value at certain relative pitch value represents the presence of maximum number of reattachment point. When the relative pitch value is less than 8 the flow cannot able to reattach before it reaches to the successive ribs due to which the thermal performance decreases. When the relative pitch value is more than 8 the reattachment per unit length is very less. Hence the heat transfer rate decreases which results lower value of Nusselt number. The Nusselt number value increase at an angle of attack $60^{\circ}$ on further increasing the angle of attack it decreases. The friction factor value increases with the increase in relative roughness pitch from 4.0 to 8.0. The friction factor value attains a maximum value at relative roughness pitch of 8.0 and decreases with further increase in relative roughness pitch. The maximum value of friction factor is obtained at an angle of attack of 90 degree, relative gap position of 0.25 and relative gap width of 1.0. The inclined ribs with gap provides higher thermo hydraulic performance as compared to the continuous rib for all value of Reynolds number. The thermo hydraulic performance is highest at relative gap position of 0.25 on further increasing the relative gap it starts to fall for all value of Reynolds number.

Bhusan et al., [13] studied the rate of heat transfer to the flowing air in the artificial roughened duct of solar heater. They reported various roughness geometries such as chamfered ribs, dimple/protrusion, corrugated ribs, $\mathrm{V}$-shaped ribs, and fins for studying heat transfer and friction 
characteristics of an artificially roughened duct of solar air heater. It has been observed that artificial roughness is a good technique to improve thermal performance of solar air heater.

Momin et al., [20] studied experimentally the effect of the geometrical parameters of the Vshaped ribs on heat transfer as well as fluid flow characteristics of rectangular duct solar air heater whose absorber plate underside is incorporated with $\mathrm{V}$-shaped ribs. The geometrical parameters such as relative roughness height $e / D_{h}$ (where $e$ is the roughness height and $D_{h}$ is the hydraulic diameter of the rectangular height) of 0.02 to 0.034 (the relative roughness height taken are 0.02 , $0.022,0.028$ and 0.034 ) and angle of attack of $30^{\circ}$ to $90^{\circ}$ (angle of attack varies as $30^{\circ}, 45^{\circ}, 60^{\circ}$ and $90^{\circ}$ ) for a fixed relative pitch of 10 have been taken for investigation of solar air heater performance. The investigation has been carried out at a Reynolds number range 2500 to 18,000 . The performance of the smooth duct solar air heater has been investigated for the same flow condition. The result of V-shaped rib roughened duct solar air heater is compared with smoother one. The Nu number increases whereas the friction factors decreases with an increase of Reynolds number. The value of Nu number and friction factor are substantially higher as compared to the smooth absorber plates. This is due to the change in fluid flow characteristics as a result of roughness associated with absorber plate which causes flow separations, reattachment and generation of secondary flow. The rate of enhancement of Nu number as compared to friction factor with the increase in Reynolds is lower. This is due to fact that the increase in relative roughness height the reattachment of free shear layer is not possible and the rate of heat transfer enhancement will not be proportional to that of friction factor. The maximum enhancement of Nu number and friction factor due to the presence of artificial roughened element has been found to be 2.30 and 2.83 times that of smooth duct for an angle of attack $60^{\circ}$. The same value of angle of attack provides maximum Nu number value and friction factor value. The thermo hydraulic performance parameters improves by increasing the angle of attack of flow as well as by increasing relative roughness height. The maximum thermo hydraulic performance has been obtained at an angle of attack $60^{\circ}$. It has been observed that at relative roughness height of 0.034 , angle of attack of $60^{\circ}$ and Reynolds number value of 17034 the v-shaped ribs solar air heater enhanced the Nu number by 1.14 times over inclined ribs where as it is enhanced by 2.30 times then the smooth plate case.

Kumar [33] studied numerically the performance of solar air heater whose bottom side of absorber plate has been incorporated with thin circular wire in v-shaped, multi v-shaped ribs and multi v-shaped rib with gap. The effect of these geometries on heat transfer, friction factor and performance enhancement has been investigated. The different turbulent model such as RNG group k-epsilon, Standard k-epsilon, Realizable k-epsilon and shear stress k-omega model have been used for analyzing the heat transfer and friction factor and their results have been compared with DittusBoelter Empirical relationship for smooth surface. The $v$-shaped rib relative roughness pitch $(\mathrm{p} / \mathrm{e})$ has been maintained at 10 . The relative roughness height $(\mathrm{e} / \mathrm{D})$ and angle of attack has been maintained at 0.043 and 60 degrees. The relative roughness width $(\mathrm{W} / \mathrm{w})$ values for multi v-shaped rib and multi $v$-shaped rib with gap has been maintained at 6 . The multi v-shaped rib and multi v-shaped rib with gap has relative roughness width(W/w), relative roughness pitch has been maintained at 6 and 10 respectively. The relative roughness height and angle of attack for both artificial roughness has been maintained at 0.043 and 60 degrees respectively. The multi v-shaped ribs with gap has been maintained its relative gap width $(\mathrm{g} / \mathrm{e})$ value at 1.0 and relative gap distance $\left(\mathrm{G}_{\mathrm{d}} / \mathrm{L}_{\mathrm{v}}\right)$ value at 0.69 . The investigation has been carried out for a duct of aspect ratio $(\mathrm{W} / \mathrm{H}) 10$. The numerical simulation has been performed using CFD code FLUENT version 6.3.26. The meshing of the computational domain has been done using commercial software GAMBIT version 2.3.16. The equation has been used for numerical simulation are mass conservation, momentum and energy equation. The uniform heat flux of $1000 \mathrm{~W} / \mathrm{m}^{2}$ has been supplied to the absorber plate. One side is supplied with uniform heat flux and the other side of the plate has been assumed to be adiabatic. At the inlet air inlet boundary condition and the outlet pressure outlet boundary condition has been used. The $v$-shaped 
rib attached to the bottom side of the absorber plate breaks and disturbs the laminar sub layer due to which the flow is wavy. The v-shaped ribs help in the development of two leading ends and single trailing ends. The formation of two leading ends and single trailing ends responsible for huge increase in heat transfer rate. The increase in relative gap width ratio $(\mathrm{W} / \mathrm{w})$ increases the number of leading ends and number of secondary flow cells. The multi v-shaped ribs with gap allow releasing secondary flow. The multi v-shaped rib with gap attached at the bottom side of the absorber plate allow the release of secondary flow along the ribs which joins the main flow to accelerates it. This helps in energizing the retarded boundary layer flow along surface resulting in enhancement of heat transfer. From the studied it has been concluded that the Numerical result and experimental result for heat transfer very close to each other. The highest value of Nusselt number has been obtained for multi v-shaped ribs with gap. The variation of Nusselt number ratio with Reynolds number for multi vshaped ribs with gap is highest. The increase in heat transfer rate in the multi v-shaped ribs with gap case is higher than that of the multi v-shaped ribs for similar cross section and operating condition due to addition of secondary flow recirculation. The Numerical results are very close to the experimental value. The highest value of friction penalty has been obtained for multi v- shaped rib with gap. The variation of friction factor ratio with Reynolds number for multi v-shaped ribs with gap is maximum. The multi $v$-shaped ribs with gap have higher thermo-hydraulic performance as compared to the multi v-shaped and $v$-shaped ribs roughened duct for the entire range of Reynolds number. The v-shaped ribs have heat transfer rate of 1.7 times the smooth duct. The multi v-shaped ribs duct have 4.7 times higher heat transfer rate than the smooth duct whereas the multi v-shaped ribs with gap heat transfer rate is 5.6 times higher than smooth duct. Renormalization k-epsilon model based results have been found in good agreement and accordingly this turbulent model is used to predict heat transfer and friction factor in the duct.

Lanjewar et al., [24] studied the heat transfer and friction factor characteristics of a rectangular duct roughened with $\mathrm{w}$ shaped $\mathrm{rib}$ on its under side on one board wall. The two different arrangements named as $w$-down and $w$-up has been taken for investigation. The $w$ shaped rib has been arranged at an inclination with the flow direction. The duct width to height ratio, relative roughness pitch of 8 and 10 has been maintained throughout the investigation respectively. The relative roughness height of 0.03375 has been taken for investigation. The angle of attack has been varied from 30-75degree to evaluate the thermal performance. The mass flow rate has been varied as per the Reynolds number range 2300-14000. With the increase in Reynolds numbers the boundary layer thickness decreases due to which the convective resistance decreases. The decrease in convective resistance increases the Nusselt number value. Hence for both the arrangement the Nusselt number value increases with the increase in Reynolds number. When the Reynolds number is low the increment of Nusselt number of roughened duct is smaller over the smooth duct and it is considered that the Nusselt number is nearly equal. At low Reynolds number the thickness of laminar sub layer increases as the flow is retarded by the roughened element and the projection of roughened element are present within the laminar sub layer. It has been observed that the maximum enhancement of Nusselt number of w-down rib of relative roughness height 0.03375 has been found to be 2.36 times than that of smooth duct for angle of attack 60 degree. The maximum enhancement of Nusselt number in case of $w$-up rib of relative roughness height 0.03375 has been found to be 2.24 times that of smooth duct for an angle of attack 60 degree. The maximum enhancement of friction factor of w-down and w-up rib at angle of attack 60 degree has been found to be 2.01 times and 2.35 times than the smooth duct respectively. The Thermo hydraulic performance of $w$ shape rib is higher as compared to the $v$ shape rib when the angle of attack is 60 degree because the $v$ shaped secondary flow induces 4 vortices and the $w$ shape rib induce 8 vortices. The boundary layer thickness in $v$ shape rib arranged in same width plate is higher as compared to the $W$ shape rib. The thermo hydraulic 
performance of w-down rib is superior as compared to the w-up and v-shape rib arranged duct. The maximum enhancement of thermo hydraulic performance in w-down rib is about 1.98 times and in w-up rib is 1.81 times.

Ghritlahre [15] studied experimentally the effect of arc shaped wire rib roughened solar air heater on heat transfer and friction factor. The investigation has been carried out for solar air heater equipped with two different orientation arc shaped wire rib. In one arc shaped roughened solar air heater the apex is upstream and in other case it is downstream flow. The studied has been carried out by maintaining the arc shaped roughness relative roughness pitch, relative roughness height, rib roughness and arc angel at $10^{\circ}, 0.0395^{\circ}, 2.5^{\circ}$ and $60^{\circ}$ respectively. To evaluate the heat transfer and friction factor of arc shaped roughened solar air heater the mass flow rate has been varied from 0.007 to $0.022 \mathrm{~kg} / \mathrm{s}$. From the investigation it has been concluded that the outlet temperature of both solar air heater increases with time and it decreases in the afternoon. The maximum outlet temperature has been obtained between 11.30 to $13.00 \mathrm{~h}$ as the solar intensity is maximum during this period. At lower mass flow rate the outlet temperature is maximum for both type of solar air heater as the residence time of fluid over heated surface will be higher. As the residence time of fluid over heated surface is more at lower mass flow rate more amount of heat will be absorbed by the fluid from heated surface of absorber plate. Hence the temperature difference of air decreases with the increase in mass flow rate. It has been concluded that the arc shaped roughened solar air heater temperature rise for apex upstream is higher as compared to the apex downstream due to proper intermixing of cold fluid with the hot fluid. The heat transfer of apex upstream flow solar air heater is enhanced with the increase in Reynolds number value and it is 2.56 times as compared to smooth solar air heater. The heat transfer rate of downstream flow has been increased by 1.81 times as compared to the smooth solar air heater. The friction factor decreases with the increase in Reynolds number because of suppression of viscous sub layers. The apex upstream flow solar air heater friction factor is 2.39 times higher than the smooth solar air heater. The downstream flow solar air heater friction factor is 2.12 times the smooth duct solar air heater, The thermo hydraulic performance of apex upstream flow is higher than the downstream flow solar air heater. Both upstream apex flow solar air heater and down stream flow solar air heater attained its maximum value at Reynolds number of 21000.The maximum thermal performance parameter of upstream apex flow is 2.09 and downstream apex is 1.59 at Reynolds number of 21000.

Prasad et al., [34] evaluated the entropy generation and second law of efficiency using the principle of second law of thermodynamics for arc shape wire roughened element incorporated solar air heater. The numerical analysis has been carried out by varying the relative roughness height from 0.0256-0.0422 and relative angle of attack from 0.33-0.66. The Reynolds number and solar intensity value have been varied from 1650-21500 and 600-1000. The studied has been carried out for a constant value of relative roughness pitch of 10 . From the investigation it has been concluded that the second law of efficiency increases up to certain range of Reynolds number. The maximum value of second law of efficiency 2.52 has been obtained at Reynolds 6460 and at solar intensity $850 \mathrm{~W} / \mathrm{m}^{2}$. On further increasing the Reynolds number the second law of efficiency starts decreasing. The losses such as optical loss, leakage loss, friction loss starts to increase on creasing the Reynolds number beyond critical value. The entropy generation increases with the increase in losses. This is responsible for lowering the value of second law of efficiency. The smooth plate solar air heater has been attained a maximum value of second law of efficiency $1.56 \%$ at Reynolds number 4398 and solar intensity $850 \mathrm{~W} / 2$. The entropy generation, entropy generation number and irreversibility increases with the increase in Reynolds number value for both roughened as well as smooth solar air heater. The minimum value of entropy generation has been obtained for relative roughness height of 0.0422 ; relative angle of attack 0.33 and relative roughness pitch 10 . Bejannumber $(\mathrm{Be})$ value decreases with 
the increase in Reynolds number. The minimum value has been obtained for relative roughness 0.044 , relative angle of attack 0.33 and pitch. This is due to entropy generation due to friction is more dominating over entropy generation due to heat transfer at this range of relative roughness, relative roughness pitch and relative angle of attack.

\subsection{Rib Formation by Machining Process}

The solar absorber plates are equipped with ribs which are generated by machining. The experimental investigations are reported in literature to study heat transfer rate and friction characteristics of rib incorporated solar absorber plate. In order to produce artificial roughness of different shape, sizes and orientation ribs have been used on absorber plate by this method as discussed in the following paragraph.

\subsubsection{Chamfered ribs}

Karwa et al., [19] investigated experimentally the performance of solar air heaters with chamfered repeated rib-roughness on the air flow side of the absorber plates. The roughened elements have a relative roughness pitch $(P / e$ where $P$ is the pitch of the rib and $e$ is the height of the roughness) of 4.58 and 7.09 while the rib chamfer angle is fixed at $15^{\circ}$. The air flow duct depths of $21.8,21.5$ and $16 \mathrm{~mm}$ have taken for investigation. The relative roughness heights $\left(e / D_{h}\right.$ where e is the roughness height and $D_{h}$ is the hydraulic diameter of the duct) for the three roughened plates used are $0.0197,0.0256$ and 0.0441 respectively. The air flow rate per unit area of the absorber plate has been varied between 0.024 to $0.102 \mathrm{~kg} / \mathrm{s}-\mathrm{m}^{2}$. The Reynolds number value will be varied from 3750 to 16350 . The roughened wall was uniformly heated with the remaining three walls insulated. The thermal efficiency of solar air heaters with artificially roughened absorber plate increased by $10 \%$ to $40 \%$ as compared to smooth surface absorber plates due to enhancement in the Nu number $50 \%$ to $120 \%$. The pumping power requirement also increased due to the increase in friction factor $80 \%$ to $290 \%$. It has been concluded that the enhancement of Nu number, friction factor and thermal efficiency wholly dependent on roughness element height. It has been observed that the solar air heater performance enhancement rate is higher at highest relative roughness height. The effective efficiency at first increases with the increase in the Reynolds number attains a maximum value. However, it starts decreasing due to substantial increase in the pumping power and it varies as the cube of the flow velocity. When the flow rate is lower and it is required to achieve the higher temperature of the air for application the solar air heater must be incorporated with roughness element of large relative roughness height. Higher pumping power is required at high flow rates. The gain in energy collection at greater relative roughness height is lower as compared to the pumping power required at high flow rates. As the pumping power required is higher than the energy gain at greater relative roughness height the smaller roughness height is maintained to increase the energy collection rate. At higher flow rate the smooth channel provides better effective efficiency.

\subsubsection{Combination of different integral rib roughness elements}

Promvonge et al., [35] investigated experimentally the effect of combined ribs and delta winglet type vortex generator in forced convection heat transfer and friction loss when the turbulent air flow through a solar air heater. The investigation has been carried out for a rectangular channel of aspect ratio 10 and height $30 \mathrm{~mm}$. The Reynolds number based on the hydraulic diameter of the channel has been varied from 5000 to 22,000 . The cross-section of the rib which is mounted on absorber plate to 
create reverse flow is an isosceles triangle. The rib height of 0.2 and rib pitch of 1.33 has been maintained throughout the experimentation. There are 10 pairs of delta winglet with its height $(b / H)$ 0.4 and transverse pitch $(\mathrm{Pt} / \mathrm{H}) 1$ has been introduced on the lower plate entrance of the tested channel. Three different angles of attack such as $60^{\circ}, 45^{\circ}$ and $30^{\circ}$ have been taken to produce longitudinal vortex flow. The combined use of ribs and delta winglets enhanced the heat transfer rate by considerable amount as compared to the smooth channel. The Nusselt number value of combined turbulator incorporated channel increases with the increase in Reynolds number. The addition of rib to the channel helps to interrupt the development of boundary layer of flowing fluid. It generates reverse or recirculating flow behind the ribs. Whereas the use of delta winglet pairs generates longitudinal vortex flow which helps to wash up the reverse flow trapped behind the ribs into the core flow. The ribs with delta winglets at higher angle of attack provides higher heat transfer rate and friction factor whereas at lower angle of attack the delta winglets pointing downstream with ribs provides best thermal performance. The ribs with delta winglets pointing upstream have higher Nusselt number value and it is about $255 \%$ higher as compared to the smooth channel. The ribs with delta winglets have higher heat transfer rate then the rib alone and it is about $50 \%$ higher. The delta winglets pointing upwards with ribs have $2-3 \%$ higher heat transfer rate as compared to delta winglets pointing down wards with ribs. The ratio of augmented Nusselt number to Nusselt number of smooth channel for delta winglets pointing upstream with ribs at angle of attack $60^{\circ}, 45^{\circ}$ and $30^{\circ}$ are $2.54,2.46$ and 2.38 respectively. Whereas for delta winglets pointing downstream with ribs the ratio of augmented Nusselt number to smooth channel Nusselt number at $60^{\circ}, 45^{\circ}$ and $30^{\circ}$ angle of attack are 2.48, 2.41 and 2.31 respectively. The combined use of rib and delta winglets has higher friction loss as compared to the rib alone as well as smooth channel. The friction factor increase rate for combined rib delta winglets is much higher as compared to the smooth channel. The delta winglets pointing upstream with ribs have higher friction factor value as compared to the delta winglets pointing downstream with ribs. The increase rate of friction factor of the combined ribs and delta winglets is in the range of 4.65 to 10.05 times over the smooth channel depending on the attack angle, the delta winglets arrangement and Reynolds number values. The friction factor of combined ribs delta winglets is $122-134 \%$ higher than the ribs arrangement alone. The ratio of friction factor of combined ribs delta winglets incorporated channel to smooth channel increases with the increase in Reynolds number value. The delta winglets pointing upstream with ribs at higher angle of attack have higher friction factor ratio value $\left(f / f_{0}\right)$ then the delta winglets pointing downstream with ribs at same operating conditions. The $\mathrm{f} / \mathrm{f}_{\mathrm{o}}$ values of delta winglets pointing upstream with ribs at angles of attack $60^{\circ}, 45^{\circ}$ and $30^{\circ}$ are $9.04,7.83$ and 6.63 respectively. The delta winglets with pointing downstream with ribs have $f / f_{0}$ values are $8.19,7.16$ and 5.88 respectively. At lower angle of attack the delta winglets pointing downstream with ribs produce less friction loss. The ribs alone provide higher heat transfer rate than the smooth channel for all Reynolds number value due to higher flow blockage as it introduce stronger reverse or recirculation flow from the rib. The heat transfer rate of rib incorporated channel is $90 \%$ higher as compared to the smooth channel. The friction factor of rib incorporated duct is very high as compared to smooth channel. The average increase in frictional loss is about 3.9 times the smooth channel. The Nusselt number of delta winglets channel with the rise in Reynolds number increases. The delta winglets with higher angle of attack have higher heat transfer than the lower angle of attack. It is due to increase in blockage to the flow with the increase in angle of attack. The friction factor of delta winglets with the increase in angle of attack increases. The use of these compound turbulator is effective for the delta winglets pointing downstream with ribs at lower attack angle or Reynolds number.

Jaurker et al., [17] studied experimentally the heat transfer and friction characteristics of ribgrooved artificial roughness on one broad heated wall of large aspect ratio duct. The experimentation 
has been carried out by varying the Reynolds numbers from 3000-21,000. The investigation has been performed by varying the relative roughness height $0.0181-0.0363$, relative roughness pitch 4.5-10.0 and groove position to pitch ratio 0.3-0.7. The effect of rib grooved artificial roughness on heat transfer and friction factor has been compared with the smooth duct for same flow conditions. The Nusselt number value of rib-grooved, rib and smooth duct increases with the increase in Reynolds number. Whereas the rib-grooved ducts have higher Nusselt number value as compared to rib and smooth duct. It is due to development of vortices in and around the groove which helps to intensify the turbulence level. The Nusselt number value increases with the increase in relative roughness pitch and attains a maximum value at relative roughness pitch of 6 . The Nusselt number after attaining the maximum value at relative roughness pitch value 6 starts to fall on further increase in relative roughness pitch. The variation of Nusselt number value at lower Reynolds number value is insignificant whereas the variation is substantial at higher Reynolds number value. At the relative roughness pitch value 6 the rib grooved duct provides higher heat transfer value at all Reynolds number value. The Nusselt number value increases with the increase in groove position to pitch ratio and attains peak value at groove position of 0.4 on further increase in groove position value the Nusselt number decreases. This effect is significant at higher Reynolds number. The Nusselt number value increases with the increases with the increase in relative roughness height. The rib-grooved duct with relative roughness pitch $(\mathrm{p} / \mathrm{e})$ of 6.0 and position of groove to pitch ratio $(\mathrm{g} / \mathrm{p})$ of 0.4 provides the maximum value of the Nusselt number. When the relative roughness pitch is 6 and position of groove to pitch ratio is 0.4 the Nusselt number value of rib-grooved duct is 2.75 times the smooth duct where as it is 1.57 times the rib duct. The friction factor decreases with the increase in Reynolds number due to suppression of viscous sub layer. The rib-grooved duct has higher friction factor as compared to rib duct. The rib grooved duct with relative roughness pitch of 6 has maximum friction factor whereas for relative roughness pitch of 4.5 has minimum value. The friction factor value increases with the increase with the relative roughness pitch and attains maximum value at relative roughness pitch value of 6 . On further increase in relative roughness value the friction factor value decreases and this effect is more pronounced at higher Reynolds number. The friction factor for rib-grooved duct with groove position to pitch ratio $(\mathrm{g} / \mathrm{p})$ of 0.4 attains maximum value and decreases on either side. The friction factor increases monotonically with the increase in relative roughness height for a given value of Reynolds number. The rib-grooved ducts with relative roughness pitch of 6 , groove position to pitch ratio of 0.4 and relative roughness height of 0.0363 attains a maximum value of the friction and it is about 3.61 times the smooth duct and 1.17 times the ribbed duct. The ribbed duct with same rib height and rib spacing the friction factor value is 3 times that of the smooth duct. The thermo hydraulic performance of rib-grooved duct is higher as compared to that of ribbed duct. The thermal hydraulic performance is maximum for all given value of Reynolds number for relative roughness pitch of 6 . The relative roughness pitch of 6 for ribgrooved the superior thermo hydraulic performance has been achieved at the groove location to pitch ratio of 0.4 .

Skullong et al., [36] investigated experimentally the turbulent flow and heat transfer characteristics in a solar air heater which is incorporated with both wavy rib and groove turbulators. The experimentation has been carried out by controlling the mass flow rate to obtain the Reynolds range of 4000-21,000. The upper wall has been supplied with constant heat flux. The recirculation flow has been introduced by wavy ribs which have been placed on the grooved channel walls. The investigation has been carried out for rib pitch to channel height ratio $0.5,1$ and 2 with single rib to channel height ratio 0.25 . The wavy ribs have been placed with the angle of attack $45^{0}$ to main flow direction. The investigation has been carried out for three different types of arrangement such as ribgroove, inline-rib groove and staggered rib-inline groove on two principal walls. From the 
investigation it has been concluded that the Nu value in rib -groove tabulators incorporated channel increases with the increase in Reynolds number because the wavy rib introduced longitudinal vortex flow which helps to enhance the degree of turbulence in the flow. It also helps to transport the core fluid to the near wall region. The vortex flow introduce by wavy rib also helps to wash up the flow trapped in the groove corner regions which normally acts as ineffective heat transfer areas. The Nusselt number value increases with the increase with the increase in Reynolds number and it has highest for inline rib-groove arrangement. The heat transfer rate of combined rib-groove tabulators is higher as compared to the single groove or inline groove alone. At rib pitch to channel height ratio 0.5 the increase in Nusselt number for using inline, staggered rib-groove upper wall are respectively in the range of $707-769 \%, 672-725 \%$ and $642-676 \%$ above the smooth channel. The rate of increase in Nusselt number value for inline groove and groove on the wall are about $168-172 \%$ and $154-159 \%$ higher than smooth channel. The rate of increase in friction factor with the increase in Reynolds for the rib tabulators is very high as compared to the groove only or smooth wall channel only. The increase rate of friction factor in rib groove tabulators is higher due to loss of dynamical pressure, reverse flow and increasing surface area. The inline rib groove has high friction factor value as compared to staggered rib -groove or rib-grooved upper wall. The friction factor value of rib-groove at rib-pitch ratio to channel height 0.5 is higher than that of groove only and it is about $38-73 \%$. The combined rib-groove arrangement has friction factor value about $14-134 \%$ times the smooth channel depending on the rib pitch to height of the channel, arrangement and Reynolds number value. The friction factor value of rib-groove at rib pitch to channel height ratio is higher than the rib pitch to channel height ratio 1 and 2 . The increase in friction factor value of inline, staggered and rib-groove in the range of $93-134 \%, 86-119 \%$ and $57-83 \%$ respectively over the smooth channel. The increase in friction factor for rib-groove and groove on one wall is about $1.8-2.3 \%$ and $1.3-1.6 \%$ over the smooth channel. The Nusselt number value as well as friction factor value increased with the increased in rib pitch to channel height ratio. The increase in Nusselt number value due to decrease in rib pitch to channel height ratio due to interruption of boundary layer development, increase in turbulence intensity of the flow and increase in mixing rate of flow. The strength of vortex flow induced at lower rib pitch ratio is higher which helps to improve the heat transfer rate further. The heat transfer rates for the rib-groove at rib pitch to channel height ratio is about $17 \%$ and $34 \%$ higher than those with rib pitch to channel height ratio 1 and 2 for all arrangement. The friction factor obtained at rib pitch to channel height ratio approximately $22 \%$ and $44 \%$ higher than that from the rib pitch to channel height ratio 1 and 2 . The rib groove at smaller rib-pitch to channel height ratio produced higher pressure drop $\left(f / f_{0}\right)$, Normalized Nusselt number value $\left(\mathrm{Nu} / \mathrm{Nu}_{0}\right)$. The range varies from $14-134$ and 4.4 -7.69 respectively depending upon the rib pitch to channel height ratio, rib arrangement and Reynolds number. The Normalized Nusselt number value augmentation tends to decrease slightly with the rise in Reynolds number. The rib-groove tabulators incorporated solar air heater produced high thermal performance of about 49-52\% higher than the groove incorporated sola $r$ air heater. The absorber plate incorporated with rib-groove at rib pitch to channel height ratio 0.5 is about 1.75 at lower Reynolds number.

\subsection{Wire Mesh or Expanded Metal Mesh Ribs}

Singh [22] studied experimentally and numerically the thermal performances of single as well as double pass porous serpentine wavy wire mesh packed bed solar air heater. The porosity level, wave length has been varied from $85 \%$ to $95 \%$ and $0.05 \mathrm{~m}$ to $0.075 \mathrm{~m}$ respectively to obtain an optimum value for geometry. The amplitude, hydraulic diameter to obtain an optimum value for geometry is varied from $0.012 \mathrm{~m}$ to $0.016 \mathrm{~m}$ and $0.025 \mathrm{~m}$ to $0.04 \mathrm{~m}$ respectively. The mass flow rate has been varied 
to obtain an optimum value of flow parameters from 0.01 to $0.05 \mathrm{~kg} / \mathrm{s}$. The thermal efficiency and thermo hydraulic efficiency increases with the increase in mass flow rate for both single and double pass solar air heater. The enhancement rate of both thermal efficiency and thermo hydraulic efficiency for single and double pass solar air heater has been insignificant at mass flow rate $0.03 \mathrm{~kg} / \mathrm{s}$. At mass flow rate $0.04 \mathrm{~kg} / \mathrm{s}$ the enhancement rate of $93 \%$ porosity packed bed double and single pass solar air heater is $80 \%$ and $68 \%$ respectively. The double pass solar air heater thermal efficiency is $18 \%$ higher as compared to the single pass solar air heater. The thermo hydraulic performance is maximum at mass flow rate $0.035 \mathrm{~kg} / \mathrm{s}$. It is about $74 \%$ and $63 \%$ for double and single pass solar air heater. The thermo hydraulic efficiency of double pass serpentine packed bed solar air heater is $17 \%$ higher than single pass. For numerical simulation ANSYS software has been used. The pressure drop increases with the increase in mass flow rate for both counter and single pass solar air heater. The maximum pressure drop has obtained at mass flow rate of $0.04 \mathrm{~kg} / \mathrm{s}$ for counter pass solar air heater and it is about $150 \mathrm{~N} / \mathrm{m}^{2}$. The maximum pressure drop of $96 \mathrm{~N} / \mathrm{m}^{2}$ has been obtained for single pass solar air heater at mass flow rate $0.04 \mathrm{~kg} / \mathrm{s}$. The maximum value of Nusselt number has been obtained for serpentine wavy configuration. The maximum value of 792 has been obtained for the porosity level $85 \%$, wave length $0.075 \mathrm{~m}$, hydraulic diameter $0.046 \mathrm{~m}$ and amplitude $0.016 \mathrm{~m}$. At hydraulic diameter $0.0835 \mathrm{~m}$ and porosity level $85 \%$ the flat one provides maximum Nusselt number of 748 . The availability of high heat transfer area, increased conduction and storage of thermal radiation results high convection heat transfer to the air which causes enhancement in Nusselt number value. The increase in porosity level further decreases the heat transfer surface area for flowing air which results poor convection heat transfer. The optimum geometrical and flow parameter to enhance the thermo hydraulic performance has been obtained on the basis of thermo hydraulic performance parameter and exergy efficiency from numerical analysis. The best thermo hydraulic performance parameter has been obtained at hydraulic diameter $=0.0835 \mathrm{~m}$, wave length $=0.075 \mathrm{~m}$, amplitude $=0.012 \mathrm{~m}$ and porosity level $90 \%$. From the exergy efficiency it has been concluded that the optimum value of mass flow rate to obtain maximum thermo hydraulic performance for serpentine configuration solar air heater is $0.03 \mathrm{~kg} / \mathrm{s}$. The enhancement is about $24 \%$ for serpentine packed bed solar air heater compared to flat packed one. From the numerical analysis it has been confirmed that the convective heat transfer rate enhances due to mixing of fluid at crest and trough regimes. There is a higher pressure drop at low packed bed porosity. The thermo hydraulic performance is maximum when the porosity level is $90 \%$, hydraulic diameter is $0.0835 \mathrm{~m}$ and when the porosity level is $85 \%$ and hydraulic diameter is $0.0474 \mathrm{~m}$. Thethermo hydraulic performance parameter is maximum due to availability of effective heat transfer area. As the pressure drop in serpentine solar air heater is higher hence in order to obtain higher thermal efficiency higher value of hydraulic diameter, lower value of amplitude and higher wave length value are preferred.

\subsection{Dimple/Protrusion Shaped Geometry}

The formation of dimples and protrusion on surfaces of absorber plate of solar air heater has been considered to be a simple and economical methodology. It is the subject of many recent experimental investigations. The heat transfer rate of dimple shape roughness is higher as compared to the smooth surfaces channel. The pressure loss penalty or friction loss usually does not increase appreciably as compared to other roughened channels. Experimental as well as numerical investigations are reported in literature to study heat transfer and friction factor characteristics of dimple/protrusion intended solar air heater.

Saini et al., [23] investigated the effect of roughness and operating parameters such as relative roughness height, relative pitch on heat transfer and friction factor for a solar air heater. The dimple 
is intended on the absorber plate. The investigation is carried out in the range of Reynolds number (Re) from 2000 to 12,000, relative roughness height (e/D where e is the roughness height and $D$ is the equivalent diameter) from 0.018 to 0.037 and relative pitch ( $\mathrm{P} / \mathrm{e}$ where $\mathrm{P}$ is the pitch of the dimple) from 8 to 12 . The roughness height taken for investigation are $0.8,1.3,1.5,1.7$ and different pitch value taken are $12,15,18 \mathrm{~mm}$ and pitch diameter taken are $0.8,1.3,1.5$ and $1.7 \mathrm{~mm}$. The Nusselt number value as a function of Reynolds number for relative pitch(P/e) values of 8,10 and 12 and for a fixed value of roughness height (e/D) of 0.0322 have been investigated. From experimental data it is confirmed that, the Nu number increases for relative roughness pitch $(\mathrm{P} / \mathrm{e})$ of 10 due to separation of the flow at the dimple, reattachment of free shear layer occurs for $\mathrm{P} / \mathrm{e}$ of 10 and maximum heat transfer coefficient occurs in the vicinity of the reattachment region. For relative pitch of 8 and 12 reattachments may not occurs hence the heat transfer coefficient value is minimum corresponds to these value of $P / e$. The larger relative roughness height $(e / D)$ produces more reattachment of free shear layer hence the maximum value of heat transfer is found to be corresponding to relative roughness height (e/D) of 0.0379 . The friction factor decreases monotonously with the increase Reynolds number for all roughness parameters. The maximum value of Nu number has been found out corresponds to relative roughness height (e/D) of 0.0379 and relative pitch (P/e) of 10. While minimum value of friction factor has been found correspond to relative roughness height $(e / D)$ of 0.02989 and relative pitch (P/e) of 10 .

Sethi et al., [37] studied experimentally the effect of dimpled shape roughness on heat transfer and friction characteristics. The dimpled shape roughness has been arranged in arc fashion or angular fashion on the absorber plate of solar air heater. The rectangular duct has maintained at an aspect ratio $(\mathrm{W} / \mathrm{H})$ of 11 , relative roughness pitch $(\mathrm{p} / \mathrm{e})$ range of $10-20$, relative roughness height $\left(e / D_{h}\right)$ range of 0.021-0.036, arc angle $(\alpha)$ range of $45-75^{\circ}$, and the Reynolds number varies from 360018000. From the investigation it has been concluded that the Nusselt number and friction factor is a strong function of relative roughness height, relative roughness pitch and arc angle. It has been observed that the Nusselt number value increase with the increase in Reynolds number. The Nusselt number value of dimple intended absorber plate is higher as compared to the smooth absorber plate. The augmentation of heat transfer coefficient in dimpled absorber plate is due to vortex generation, flow separation and vortex shedding. The Nusselt number value for relative roughness pitch 10, 15 and 20 for a fixed arc angle of 45 degree and relative roughness height of 0.036 increases monotonously with the increase in Reynolds number. The Nusselt number value is maximum at relative roughness pitch value of 10 and it is due to separation of flow for dimple and reattachment of free shear layer. With the increase in relative roughness pitch value the number of dimples decreases or the distance between the dimple increases due to which the formation of vortices reduced which adversely affect the heat transfer rate. The Nusselt numbers value for arc angel $45^{\circ}$, $60^{\circ}$ and $75^{\circ}$ at a fixed value of relative roughness pitch of 10 and relative roughness height of 0.036 varies with respect to Reynolds number. The Nusselt number value increases with the increase of arc angle up to 60 degree beyond that value it starts decreasing. The Nusselt number value increases with the increase in Reynolds number value by varying the relative roughness height from 0.021-0.036 and fixing relative roughness pitch at 10 and arc angle at 45 degrees. The Nusselt number value is maximum at relative roughness height 0.036 . Higher the relative roughness height more will be the reattachment of free shear layer which introduced strong secondary flow and stronger the secondary flow higher will be the heat transfer rate. The friction factor decreases with the increase in Reynolds number due to suppression of viscous sub layer. The friction factor of roughened absorber plate is always higher as compared to the smooth absorber plate. The maximum value of friction factor has been introduced for relative roughness pitch of 10 due more number of reattachment points. With the increase in relative roughness pitch the number of dimple decreases hence friction loss 
decreases. The friction factor at arc angle 60degree is maximum whereas at arc angle 45 degree the friction penalty is minimum. When the arc angle is 60 degrees the maximum number of dimple comes in contact of the flowing fluid. The friction factor value increases with the increase in relative roughness height and it attains a maximum value at relative roughness height 0.036 . It is due to formation of strong recirculation region which creates strong secondary flow and the power consumption of flow air increases rapidly.

Yadav et al., [38] studied experimentally the heat transfer and friction characteristics of turbulent flow of air passing through a rectangular duct which is roughened with circular protrusion in angular arc fashion. The heat transfer and friction factor characteristics has been investigated for the Reynolds number ranges from 3600 to 18,100 and for three different value of relative roughness pitch $(P / e)$ such as 12,18 and 24 . The relative roughness height $(e / D)$ ranges from 0.015 to 0.03 and three different arc angle of protrusion ( $\alpha$ ) arrangement such as $45^{\circ}, 60^{\circ}$ and $75^{\circ}$ have been taken for investigation. The Nusselt number value for a given roughness parameter (e/D) and arc angle( $\alpha)$ for all relative roughness pitch $(\mathrm{P} / \mathrm{e}) 12,18$ and 24 increases monotonously with the increase in Reynolds number. The value of Nusselt number of protruded absorber plate is higher than smooth one for a given Reynolds number. The heat transfer enhancement in protruded absorber plate is due to flow impingement, formation of vortex on both sides of protrusion and also due to flow separation. The flow impingement occurs on the front side of the protrusion while vortex is generated due to hindrance created by protrusion. The Nusselt number value decreases for the Reynolds number range $3600-18,100$ with the increase in relative roughness pitch $(\mathrm{P} / \mathrm{e})$ at a constant relative roughness parameter $(e / D=0.03)$ and angle of attack $\left(\alpha=60^{\circ}\right)$. It has been concluded that among three relative roughness pitch 12, 18 and 24 the maximum Nusselt number value has been obtained at relative roughness pitch 12 . With the increase in relative roughness pitch the number of reattachment point decreases on the protruded absorber plate. From the investigation it has been concluded that in order to increase the heat transfer rate the relative roughness pitch must be decreased. It has been observed that the relative roughness pitch should not be below 8 because with the decrease in relative roughness pitch value further the reattachment point cannot able to form which leads to reduction of heat transfer rate. The Nusselt number value increase up to arc angle $60^{\circ}$ by further increasing arc angle the Nusselt number starts to decrease. This is due to the vortex legs originated at the former protrusion affects the downstream protrusion located in the angular shape direction. With the increase in arc angle further the number of protrusion on the absorber plate increased and the distance between the protrusion in the diagonal direction decreased hence it unable to break laminar sub layer as a result of heat transfer rate decrease. The friction factor decreases with the increase in Reynolds number. The friction factor of protruded solar absorber plate is higher than smooth solar absorber plate. The friction factor is minimum for relative roughness parameter 0.015 and it is maximum for relative roughness parameter 0.03 , relative roughness pitch 12 and arc angle $60^{\circ}$ due to change in fluid flow characteristics.

Bhusan et al., [39] investigated the thermal and thermo hydraulic performance of smooth absorber plate as well as roughened absorber plate solar air heater using mathematical model. The solar air heater has been roughened with the formation of protrusion on solar air heater. From the investigation it has been concluded that the thermal efficiency at the entire range of temperature rise parameter is maximum at short way length 31.25. The thermal efficiency decreases for a given value of short way length with the increase in temperature rise parameter. The thermal efficiency for the entire range of Reynolds number is maximum at short way length 31.25 due to high heat transfer coefficient value. The heat transfer coefficient value is maximum due to main flow impingement, flow separation and vortex generation on both sides of protrusion. For the entire range of temperature range rise parameter, the thermal efficiency is maximum for long way length 31.25 . It 
has been observed that the thermal efficiency decreases with the rise in temperature rise parameter for a given value of long way length. For all range of Reynolds number, the thermal efficiency is maximum at long way length 31.25 due to higher heat transfer coefficient value. The heat transfer coefficient value is maximum due to flow separation at protrusion and reattachment of free shear layer in between two protrusions long way direction. The thermal efficiency is maximum at the entire range of temperature rise parameter for relative print diameter 0.294 . The thermal efficiency for a given value of relative print diameter decreases with the rise in temperature rise parameter. The maximum value of thermal efficiency has been obtained due to increase in heat transfer coefficient at relative print diameter 0.294 for all range of Reynolds number. The heat transfer coefficient is maximum due to effective short way and long way distance between the protrusions which can break laminar sub layer along the passage of air flow. The thermal efficiency of roughened solar air heater is 2.3 times higher than the smooth plate solar air heater. The effective efficiency value is maximum for short way length 31.25 for the entire range of temperature rise. At a given value of short way length, the effective efficiency increases with the increase in Reynolds number attained the maxima and then it starts decreasing. However, in case of smooth plate solar air heater with the increase in Reynolds number the effective efficiency increases. For the entire range of temperature rise parameter the effective efficiency at long way length 31.25 attains a maxima value with the increase in temperature rise parameter and then starts to decreases on further increase in the temperature rise parameter. The effective efficiency of smooth plate solar air heater decreases with the increase of temperature rise parameter. The effective efficiency for the entire range of Reynolds number attains a maximum value at long way length 31.25 and then starts decreasing with further increase in Reynolds number. However, for smooth solar air heater effective efficiency is found to increase for entire range of Reynolds number. At the entire range of temperature rise parameter effective efficiency attains a maxima value for relative print diameter 0.294 . At a given relative print diameter the effective efficiency attains a maxima value then starts to decrease. The effective efficiency of smooth solar air heater decreases for the entire range of temperature rise parameter. The effective efficiency attains a maximum value at relative print diameter 0.294 with the rise in Reynolds number. The effective efficiency for a given relative print diameter increases with the rise in Reynolds number attains a maximum value then starts decreasing with further increase in Reynolds number. However, for smooth solar air heater effective efficiency is found to increase for the entire range of Reynolds number. For the temperature rise parameter lower than $0.0025 \mathrm{~m}^{2} \mathrm{k} / \mathrm{W}$ the relative short way length of 37.5 represents the optimum value for any insolation. For the temperature rise parameter higher than $0.005 \mathrm{~m}^{2} \mathrm{k} / \mathrm{W}$ the relative short way length of 27 represents optimum value for any insolation. For temperature rise parameters less than $0025 \mathrm{~m}^{2} \mathrm{k} / \mathrm{W}$ the relative long way length 0 33 represents optimum value for any insolation. For temperature rise parameter higher than $0.0035 \mathrm{~m}^{2} \mathrm{k} / \mathrm{W}$ relative long way length of 31.25 represents optimum value for any insolation. For temperature rise parameter range less than $0025 \mathrm{~m}^{2} \mathrm{k} / \mathrm{W}$ the relative print diameter value of 0.022 represents optimum value for any insolation.

\subsection{Fins and Baffles/Vortex Generator}

Pakdamann et al., [40] investigated experimentally the different thermal characteristics of natural convection solar air heater which has been incorporated with longitudinal fin array. From the investigation it has been concluded that heat transfer of solar air heater can be enhanced by the attachment of longitudinal rectangular fins array. The heat transfer enhancement in solar air heater is due to the increase in additional surface area as well as due to the mixing effect introduced by these elements. It has been observed that due to the increment of heat transfer surface area by $66 \%$ 
the increase in heat transfer rate is about $20 \%$. The heat transfer rate is also negligible dependency of inclination angel of these devices. The thermal efficiency of finned incorporated solar air heater increases due to the increase in solar radiation intensity as well as with the increase in ambient temperature and the maximum value obtained at noon time. It has been evident from experimental results that the effect of solar radiation intensity on the thermal performance of the system to be 79 times greater than the effect of the ambient temperature.

Sawhney et al., [41] investigated experimentally the heat transfer and friction factor of solar air heater absorber plate roughened with wavy-up delta winglet vortex generators. The solar air heater absorber plate has been supplied with constant heat flux. The Reynolds number ranges based on hydraulic diameter have been taken for investigation varies from 4000-17,300. The investigation has been carried out for delta winglet wave number $(\varnothing) 3,5$ and 7 . The relative longitudinal pitch $(P / H)$ at which the investigation has been carried out varied as $3,4,5$, and 6 respectively at a single angle of attack $(\alpha) 60^{\circ}$. The investigation has been carried out for two different type of arrangement such as in line and staggered. The heat transfer rate from the absorber plate which has been incorporated with various types of winglets has been studied and compared it with the heat transfer rate of smooth solar absorber plate. On comparing it has been found out that the heat transfer rate of winglets incorporated solar air heater is higher as compared to the smooth one. The heat transfer rates of 3 wave winglets for longitudinal pitches $(P / H)$ 3,4,5,6 have been calculated. The studied of Nusselt number variation with respect to Reynolds number for 3 winglets wave at longitudinal pitch $(\mathrm{P} / \mathrm{H})$ $=75,100,125,150$ and smooth absorber plate have been carried. From the investigation it has been observed that the highest value of Nusselt number obtained at relative longitudinal pitch value equal to 3 because with the increase in longitudinal pitch the number of winglets pair used will be decreased and the strength of the vortex reduced and it break down. With the decrease in relative longitudinal pitch the number of winglets pair used increased and more vortices motion results from the winglets pairs leading to higher Nusselt number. Due to shorter relative pitch the vortex generated at a low retains sufficient strength as it transverses longitudinally along the flow till it interacts with the vortices generated by the next row both being co rotating on superimposition produce a stronger vortex. This leads to higher Nusselt number value. At wave number $(\varnothing)=3$ the Nusselt number increases and it reaches to a maximum value at $\varnothing=5$. Further increase in wave number decreases the Nusselt number because with the increase in wave number the distance between the wave will be decreased and the vortex generated by one will be cancelled by other. Due to this cancellation of vortex the Nusselt number is reduced by increasing the wave number. The maximum enhancement in the Nusselt number is found to be 3.87 times the smooth duct for winglets at $P / H=3$ and $\varnothing=5$. The enhancement of Nusselt number is greater at lower Reynolds number. The value of friction factor decreases with the increases in Reynolds number for all value of $\mathrm{P} / \mathrm{H}$. With the increase in longitudinal pitch the friction factor value decreases whereas with the decreases in $\mathrm{P} / \mathrm{H}$ value as the number of winglets used increases as a result of which friction factor increases. Greater the number of winglets pair used more will be the obstruction to flow and more is the friction factor. The friction factor enhancement is highest for relative longitudinal pitch of 3 and number of waves 7 and it is 10.9 times the smooth duct. The thermal enhancement factor tends to increases with the decreasing longitudinal pitch due to increase in the higher rate of heat transfer at lower relative longitudinal pitch. The thermal enhancement factor of wavy delta winglets with wave number 5 is found to be about $116.7 \%$ and $104.1 \%$ higher than the wavy delta winglets with wave number 3 and 7. At wave number 5 and Reynolds number 4000-17,300 thermal enhancement factor varied between 1.46, 2.09, 1.32 and 1.84 and $1.19,1.69,1.02$ and 1.46 for longitudinal pitch $3,4,5,6$. The thermal performance factor of 2.09 is obtained at relative longitudinal pitch value of 3 and wave number 5 . The higher Nusselt number value has been obtained for inline arrangement. 
Karim et al., [42] studied the performance of three different type of solar collector named as flat, finned and $\mathrm{V}$-corrugated $\left(60^{\circ}\right)$ under different mass flow rate condition. The performance of the three different solar air heater is investigated for the mass flow rate of $0.056 \mathrm{~kg} / \mathrm{m}^{2} \mathrm{~s}, 0.0387 \mathrm{~kg} / \mathrm{m}^{2} \mathrm{~s}, 0.0317$ $\mathrm{kg} / \mathrm{m}^{2} \mathrm{~s}, 0.0248 \mathrm{~kg} / \mathrm{m}^{2} \mathrm{~s}$ and $0.0154 \mathrm{~kg} / \mathrm{m}^{2} \mathrm{~s}$. The collectors are also investigated in double pass mode to investigate the extent of improvement in efficiency that can be achieved without increasing the collector size or cost. During each test the inlet temperature of air and flow rate are remains constant from the experimentation data it is evident that the flat plate collector with single pass is least efficient and the V-groove collector provides highest efficiency. The V-groove collectors are also structurally stable. The $\mathrm{V}$-corrugated collector has $7 \%$ to $12 \%$ higher efficiency than the flat plate collector and $5 \%$ to $9 \%$ more efficient than the finned collectors. The $\mathrm{V}$-corrugated collector can absorb greater quantity of solar radiation as compared to the flat plate and finned collectors of equal absorptivity. The absorption capacity of v-corrugated solar collector is higher because of multiple reflection and absorption of incident radiation. The efficiency of all three air collector is a strong function of mass flow rate of air. The efficiency is enhanced with flow rates and tends to saturate beyond a flow rate $0.056 \mathrm{~kg} / \mathrm{m}^{2} \mathrm{~s}$ because the outlet temperature of air is decreased. The optimal ranges of flow rate suitable for drying of agricultural products are in between 0.025 to $0.035 \mathrm{~kg} / \mathrm{m}^{2} \mathrm{~s}$. Since the V-corrugated collector provides better efficiency in both the single and double pass operation and also structurally stable this collector is considered predominantly useful for drying application.

Choudhuary et al., [26] studied the effects of air flow velocity and air channel depths on the air temperature increment, the collector efficiency and pressure drop experienced by the flowing air for different channel lengths and different specific mass flow rates of air. The studied have been carried out using two different type of solar air heater named as corrugated and plane solar air heater. The investigation is carried out for 5 different configurations. Type - 1 in which the absorber plate is corrugated and the cover plate is plane, Type - 2 the lower side of the cover plate is corrugated and absorber plate is plane, Type - 3 the cover plate as well as absorber plate is plane, Type - 4 the absorber plate is corrugated and the back plate is plane, Type - 5 the cover plate, absorber plate and back plate is plane. The Type - 1 configuration thermal performance has been higher as compared to others. When the mass flow rate increases the efficiency increases the increase is rapid at low flow rate whereas increases in efficiency less rapid at higher flow rates. The efficiency for a fixed collector length and specific air flow rate increases initially rapidly with the increase in air velocity and then increases slowly. The increases in efficiency with the increases in air velocity for a fixed collector length and flow rate increases in shallow depth air channel. The dependence of efficiency on air velocity is more predominant at higher mass flow rate then lower flow rate. The increment of flowing air temperature decreases the efficiency of the solar air heater. The efficiency of solar air heater at higher flow rate as well as lower flow rate and temperature rise of flowing air depends on the velocity of the air. The rise of temperature of the flowing air can be increased by decreasing the air flow rate per unit area and the loss in efficiency at lower flow rates can be avoided by increasing the channel depth for a fixed specific air flow rate and a fixed air velocity the system efficiency decreases with increase in the air channel depth. For fixed specific air channel length, the efficiency increases with increased air velocity or with decreased in air channel depth. With the increase in mass flow rate for a specific length of the collector, air velocity and with decrease in air channel depth the pressure drop increases.

Kabeel et al., [43] carried out a comprehensive literature review on various heat transfer improvement techniques, design configurations and applications of different types of solar air heater. From the literature review it has been concluded that performance of solar air heater can be enhanced by the use of various types of fins such as longitudinal fins, corrugated fins and fins 
attached with baffles. It has been concluded that the selection of height, width of these roughened element is an important factor to achieve maximum benefits of these elements. The efficiency of the fin attached solar air heater can reach to an approximate value of $64 \%$ for the double pass solar heater. The recycling of flowing air has been considered as one of the best techniques to enhance the performance of solar air heater. The solar air heater incorporated with baffles also increased the thermal performance of solar air heater. From the survey it has been observed that the $\mathrm{v}$-corrugated solar air heater is most efficient as compared to flat plate solar air heater. The use of artificial roughened element on the solar air heater also increased the Nusselt number and friction factor for a wide range of Reynolds number. It has been concluded from literature survey that the use phase change material, packing bed for storing heat and by applying proper coating over the surface of the absorber plate helps to improve the thermal performance of solar air heater.

Kumar et al., [44] carried out a comprehensive literature review on various small height artificial roughness incorporated solar air heater to enhance the thermal performance. The thermal performance of various artificial roughness geometries such as ribs, dimple, baffles, wire mesh, delta winglets etc have considered during the literature survey. From the literature survey is has been concluded that by the addition of artificial roughness enhance the heat transfer rate simultaneously it also increases the pressure penalty. It has been concluded that the maximum heat transfer enhancement is obtained for dimple incorporated solar air heater which is followed by multi-v shaped rib with gap and multi v-shaped rib without gap. The maximum heat transfer enhancement has been obtained for dimple imprinted solar air heater and it is about 7.58. The heat transfer enhancement for multi v-shaped rib with gap is obtained as 6.74 and without gap has been recorded as 6 . From the literature survey it has been concluded that the friction factor for dimple imprinted solar air heater has been recorded as 4.68. The friction factor of multi v- shaped rib with gap has been recorded as 6.37 and multi v-shaped rib without gap recorded as 5 . The value of thermo hydraulic performance reported by the various investigator varies from 1.38 to 2.45 .

Kabeel et al., [45] investigated experimentally the thermal performance of flat, finned, and Vcorrugated $\left(60^{\circ}\right)$ single glass cover, single pass solar air heater. The influence of mass flow rate of air on the exit temperature and thermal efficiency of the heater has been studied by taking three different value of mass flow rate such as $0.062,0.028$ and $0.009 \mathrm{~kg} / \mathrm{sec}$. The thermal efficiencies of $\mathrm{V}$-corrugated solar heater obtained at the mass flow rate $0.062,0.028,0.009 \mathrm{~kg} / \mathrm{sec}$ are $50 \%, 43.2 \%$ and $22.2 \%$ respectively. For finned and flat solar heater thermal efficiencies obtained are $41.8 \%, 39.1 \%, 18.6 \%$ and $40.6 \%, 32 \%, 14.4 \%$ when the mass flow rate are $0.062,0.028$ and 0.009 $\mathrm{kg} / \mathrm{sec}$ respectively. The $\mathrm{V}$-corrugated collector is found to be one of the most efficient collectors and the flat plate collector is one of the least efficient collectors. It has been concluded that the $\mathrm{V}$ corrugated solar air heater shows thermal efficiency of about $8 \%$ to $14.5 \%$ and $6 \%$ to $10.5 \%$ higher than the flat plate and finned plate solar heater for mass flow rate $0.062 \mathrm{~kg} / \mathrm{s}$. The exit temperature of v-corrugated solar air heater which provides best performance increases by $5^{\circ} \mathrm{C}$ when the mass flow rate is $0.062 \mathrm{~kg} / \mathrm{sec}$. The exit temperature of $v$-corrugated solar air heater $3.5^{\circ} \mathrm{C}$ more than the flat plate and finned plates when the mass flow rate is $0.062 \mathrm{~kg} / \mathrm{sec}$. The convection heat transfer coefficient of v-corrugated type solar air heater is 1.64 times higher than the flat type solar air heater and 1.36 then the of finned type solar heater at flow rate of $0.062 \mathrm{~kg} / \mathrm{sec}$. The $\mathrm{V}$-corrugated solar air heater has higher heat transfer area than the finned and flat surface solar air heater. The turbulence inside the air channel increases due to which the collection of useful heat by the V-corrugated solar heater is higher as compared to finned and flat solar air heater.

Hachemi et al., [46] studied the enhancement of thermo physical properties of air which is used as a fluid for heat transfer in solar air heater using fins. The effect of fin dimension as well as distance between two consecutive fins on heat transfer rate has also been studied. To enhance the heat 
transfer rate of air fully developed flow has been created by soldering rectangular type fin under the absorber plate which is arranged in staggered fashion. Due to this arrangement the fluid flow under goes contraction followed by expansions which creates fully developed turbulent flow and increases the thermal heat transfer between the absorber plate and the air. The use of fin enhances the surface area for heat transfer due to which there is an appreciable improvement of the thermal performance of solar air heaters as compared to those solar air heaters with plane absorber plate. The staggered arranged solar air heater efficiency increases as compared to plane absorber plate. With the decrease in distance between the consecutive fin the heat transfer rate increases. The thermal heat performance for a specific mass flow rate $50 \mathrm{~kg} / \mathrm{h}-\mathrm{m}^{2}$, length of the fin $10.0 \mathrm{~cm}$ and distance between the two consecutive staggered rows fin $2.5 \mathrm{~cm}$ is $38.6 \%$ for plane absorber plate and for finned absorber plate is $75.5 \%$ and the temperature rise increases for the plane absorber plate is $27.7^{\circ} \mathrm{C}$ and for finned absorber plate solar heater $54.1^{\circ} \mathrm{C}$.

Kabeel et al., [47] studied the effect of shape factor of triangular type absorber plate solar air heater and longitudinal fins type solar air heater. The flow passes over and under the absorber plate in case of triangular type solar air heater. Whereas the flow passes between the absorber plate and first glass cover in case of longitudinal type solar air heater. The collector efficiency factor is a function of absorber shape factor. The collector efficiency increases with the increase in the absorber shape factor. The increment rate of collector efficiency is higher up to the shape factor 3 then the increment rate on further increasing shape factor is very small. The collector efficiency factor for the triangular type solar air heater is higher than the longitudinal fins type solar air heater. The heat transfer rate to the flow as well as the pressure loss penalty in the solar air heater increases with the increase of the triangular angle of the triangular collector. The optimum angle of the triangular collector varies from $50^{\circ}$ to $60^{\circ}$.

Akpinar et al., [48] investigated the thermo hydraulic performance of solar air heater which is incorporated with 3 different types of obstacles. The obstacles incorporated to absorber plates are categorized as Type - I, Type - II, and Type - III and the solar air heater without obstacles is considered as Type - IV. Type - I obstacles is triangular obstacles of $5 \times 5 \mathrm{~cm}^{2}$ dimension which are situated on the absorber plate at $10 \mathrm{~cm}$ intervals with $3.5 \mathrm{~cm}$ distance between the successive lines. Type - II obstacles are the leaf shaped obstacles of $5 \times 5 \mathrm{~cm}^{2}$ dimension which are situated on the absorber plate at $10 \mathrm{~cm}$ intervals with $3.5 \mathrm{~cm}$ distance between the successive lines. Type - III is the rectangular obstacles of $10 \times 10 \mathrm{~cm}^{2}$ dimensions which are situated at $2.5 \mathrm{~cm}$ intervals with at a $45^{0}$ angle on the absorber plate. The investigation is carried out for two different mass flow rate $0.0074 \mathrm{~kg} / \mathrm{sec}$ and 0.0052 $\mathrm{kg} / \mathrm{sec}$. From the experimental data recorded it is evident that efficiency of the solar air collector depends significantly on the solar radiation, surface geometry of the collector and extension of the air flow lines. The intensity of solar insolation is highest at 12.30PM. The highest daily solar radiation is obtained as $1016 \mathrm{~W} / \mathrm{m}^{2}$. The intensity of radiation increases in the morning to a peak value of 1016 $\mathrm{W} / \mathrm{m}^{2}$ at noon and starts decreases in the afternoon. The highest intensity of radiation absorbed by Type - II type solar absorber plate and lowest is Type - IV type solar absorber plate. The highest temperature increases occurred at periods of 12.00-14.00. The maximum difference temperature increase through the four types of solar air heaters (I-IV) was $45.9,50.5 .44 .1$ and $33.1^{\circ} \mathrm{C}$ for $0.0074 \mathrm{~kg} / \mathrm{sec}, 47.4,55.4,48.5$ and 38.3 for $0.0052 \mathrm{~kg} / \mathrm{sec}$. The highest temperature difference increases occurred through Type - II whereas the lowest enhancement through Type - IV. The efficiency of the collectors improves with increasing mass flow rates due to an enhancement of heat transfer to the air flow. The highest temperature difference enhanced the efficiency and reduces the overall heat loss. From this it is concluded that the obstacles ensure a good air flow over and under the absorber plate create turbulence and reduced the dead zones in the collector. 
Ong [49] studied theoretically thermal performance of four different types of flat plate solar air heater and compared the result with the experimental result. The surface and air temperature have been predicted theoretically at Reynolds number ranges 3300 to 40,000 for four different types of solar collectors. The matrix inversion solution procedure to obtain theoretical prediction of the performance has been used. The various types of solar air heater used for the investigation were Type - Ila, Type - IIb, Type - III and Type - IV. Type - Ila solar collector was a single air flow between absorber and bottom plates with no bottom insulations. Type - Ilb was a single channel design with single air flow between absorber and bottom plates with bottom insulation provided. Type - III was a double design with single air flow between absorber plates and with bottom insulation provided. Type - IV was a double channel design with double air flows between top glass and absorber and bottom plates and with bottom insulation provided. It has been concluded from the studied that temperature decreases with the increase in flow rates. The absorber plate exhibited the highest temperature with glass being at lowest. The temperature difference between the air streams and the bottom plate becomes less with the increase in air flow rate. The result showed that agreement between experimental data and predicted results was within $5^{\circ} \mathrm{C}$.

Yeh et al., [50] investigated experimentally and theoretically the effect of parallel barrier on collector efficiency and compared the collector efficiency of solar collector without barrier. The collector efficiency has been investigated for three different mass flow rate $0.0214 \mathrm{~kg} / \mathrm{sec}$, $0.0161 \mathrm{~kg} / \mathrm{sec}$ and $0.0107 \mathrm{~kg} / \mathrm{sec}$ and for two different solar radiation intensity $1100 \mathrm{~W} / \mathrm{m}^{2}$ and 830 $\mathrm{W} / \mathrm{m}^{2}$. The barrier converted the solar collector into two sub collector. The surface area of the solar collector has been maintained constant at $0.42135 \mathrm{~m}^{2}$ (Where $L$ (Length) $=26.5 \mathrm{~cm}, \mathrm{~B}$ (width) $=159 \mathrm{~cm}$ ) but the location of the barrier has been changed. The changes in the location of the barrier provided in nine different ratio of $B_{1} / B$ such as $1 / 6,1 / 5,1 / 4,1 / 3,1 / 2,2 / 3,3 / 4,1 / 5$ and 5/6. The barrier used to divide the flow channel was thin and thermally separated. From the investigation it is concluded that with the increase in $B_{1} / B$ the collector efficiency increased and it was maximum at $B_{1} / B=0.5$ and with the increase in $B_{1} / B$ further it decreased. More parallel barriers placed inside the collector with the same distance between any two of them, the higher collector efficiency can be obtained by increasing either the aspect ratio (L/B) or barrier number which decreases the cross-sectional area of the air duct and thus increases the velocity of air flow as well as the convection heat transfer rate from the surface of the absorbing plate to the flowing air. The collector efficiency is higher at higher flow rate and it is maximum at $\mathrm{B}_{1} / \mathrm{B}=0.5$. The result showed agreement between experimental and predicted results.

Chabane et al., [51] studied experimentally the thermal performance of single pass solar air heater incorporated with five numbers of longitudinal fins. The comparison of solar collector thermal performance with fins and without fins has been performed. The longitudinal fins have been attached at the bottom side of the absorber plate which helps to maintain uniform flow in a channel. The effect of mass flow rate on the outlet temperature of flowing air, heat transfer rate in the thickness of the solar collector as well as thermal efficiency have been studied. The investigation has been carried out for two air mass flow rate of 0.012 and $0.016 \mathrm{~kg} / \mathrm{s}$. From the investigation it has been concluded that the efficiency of the solar collector is a function of solar radiation intensity, mass flow rate, surface geometry of the collector and the fins attached at the back side of the absorber plate. The efficiency of the solar collector increases with the increase in solar radiation intensity at mass flow rate 0.012 and $0.016 \mathrm{~kg} / \mathrm{s}$. It is mainly due to higher heat transfer rate to the flowing air. The collector efficiency and outlet air temperature has been achieved at tilt angle 45 degree. The lowest collector efficiency has been obtained from the collector without fins. The efficiency of the collector has been increased significantly when the mass flow rate varied from 0.012 to $0.016 \mathrm{~kg} / \mathrm{s}$ due to changes in flow condition from laminar to turbulent. The slope of the efficiency curves decreases with the increase in mass flow 
rate due to decrease in heat loss coefficient. The maximum thermal efficiency of the solar collector with fins has been obtained at mass flow rate of 0.012 and $0.016 \mathrm{~kg} / \mathrm{s}$ is $40.02 \%$ and $51.50 \%$. Whereas the maximum thermal efficiency of the solar collector without fins at mass flow rate $0.012 \mathrm{~kg} / \mathrm{s}$ is $34.92 \%$ and at $0.016 \mathrm{~kg} / \mathrm{s}$ is $43.94 \%$.

Behura et al., [52] studied experimentally thermal performance of three side glass cover with three side artificially roughened solar air heater for fully developed turbulent flow condition. The artificially roughened solar heater heat transfer rate, friction factor and pumping power requirement has been compared with smooth solar air heater. At the relative roughness height fixed value of 0.0247 the Nusselt number increases with the increase in Reynolds number for relative roughness pitch value of 10,15 and 20 . The Nusselt number value attains a maximum value when the relative roughness pitch value is 15 for three side glass cover with three side roughened collector. The increase rate of Nusselt number of roughened collector is rapid as compared to the smooth collector. At Reynolds number 9806 the three side glass cover with three side roughened collector has Nusselt number of 58, 62 and 66 for the relative roughness pitch value of 20,15 and 10 respectively. At this given condition the Nusselt number value of smooth collector is 32 . At a fixed relative roughness pitch value 10 with the increase in relative roughness height and Reynolds number value the Nusselt number value increases in a rapid manner as compared to smooth one. The Nusselt number value is maximum at relative roughness height value of 0.0247 . At Reynolds number 9806 the three side roughened collector has Nusselt number value of 56,61 and 64 for relative roughness height of $0.0135,0.0025$ and 0.0247 respectively. The Nusselt number value of smooth collector is 30 at Reynolds number value of 9806 . For a fixed value of relative roughness height of 0.0247 with the increase in Reynolds number the friction factor decreases with the decrease in value of relative roughness pitch for roughened collector. The friction factor is highest for relative roughness pitch of 10. The friction factor with the increase in relative roughness height increases. The friction factor is maximum for relative roughness height of 0.0247 . The friction factor decreases with the increase in Reynolds number for three sides glass cover with three side roughened collector and smooth collector. Heat transfer increment factor has been varied in between $0.378-0.487$ by varying relative roughness height and relative roughness pitch.

Gao et al., [53] studied numerically the natural convection inside the channel between the flat plate cover and the sine wave absorber in a cross-corrugated solar air heater. The effect of major system parameters such as $\Delta T$ the difference in average temperature between the cover and absorber,' $A^{\prime}$ the characteristics height ratio of the channel which is defined as the ratio of channel height to the amplitude height of the wave like absorber, $L$ the characteristics geometric ratio which defined as the ratio of fourth of the wave length to the amplitude height of the absorber and $\theta$, the angle of inclination of the heater on the natural convection have been investigated numerically. The flow inside the channel between the cover and the absorber plate is natural convection and the flow is assumed to be laminar. The Boussinesq fluid region is said to be two dimensional. The study has been carried out at steady state and the fluid is assumed to be incompressible. No heat source or sink inside the channel and the radiation gain or loss is assumed to be negligible. The fluid properties are assumed to be constant except density because of the density difference the buoyancy force effect becomes significant which provide driving force for natural convection. The no slip boundary condition $(u=0, v=0)$ has been applied at the solid boundaries of the channel. Uniform temperature boundary condition has been applied to the flat plate cover and the wave like absorber plate. Adiabatic boundary conditions are applied to the two sides of the channel. The governing equation used for numerical analysis are continuity equation, Navier stokes equation and energy equation. It is concluded from the investigation that the heat transfer coefficient $\mathrm{h}$ increases with the $\Delta T$ value. The heat transfer coefficient value increases linearly and sharply at $\Delta \mathrm{T}\left\langle 10 c^{0}\right.$. The trend after that is 
asymptotic. The heat transfer value increases with $A<1.5$ but beyond this value the heat transfer coefficient starts to fall and this decreasing rate is almost linear. In order to minimize the heat loss due to natural convection from the channel the value of $A$ should be greater than 2 . The heat transfer coefficient value increases sharply with $0.25 \lambda$ /a when it is less than 0.8 but it starts to decrease sharply thereafter and the decreasing rate slow down gradually. To minimize the convection heat loss from the channel the $\left(\frac{0.25 \lambda}{\mathrm{a}}\right)$ should be larger than 1 . The heat transfer coefficient value increases with $\theta$ slowly when $\theta<50$ and when $\theta=60^{\circ}$ it begins to decreases gradually.

Mohammadi et al., [54] investigated the influence of fins and baffles attached over the absorber plate on performance of the upward type single pass solar air heater. The performance evaluation has been carried out by investigating outlet temperature of flowing air, efficiency and effective efficiency. To evaluate the overall performance, the assumption has been taken such as (i) the mathematical formulation are written in one dimensional and under steady state, (ii) the heat capacities of glass cover absorber plate and insulation is negligible, (iii) the air leakage from the solar air heater and the edge losses are negligible, (iv) the air dose not absorb solar radiation, (v) the temperature of the flowing air varies only in the direction of flow. The variation of outlet temperature of flowing air with the increase in mass flow rate for different width baffles for solar radiation intensity $700 \mathrm{~W} / \mathrm{m}^{2}$ and $1000 \mathrm{~W} / \mathrm{m}^{2}$ has been evaluated. The outlet temperature of flowing air increases with the increase in width of the baffles for all mass flow rates. The outlet temperature of the air increases as compared to the conventional solar air heater due to increase in heat transfer rate from the absorber plate to the air. The percentage of increase in outlet temperature of air decreases slightly with the increase in width of the baffles. At solar intensity radiation $700 \mathrm{~W} / \mathrm{m}^{2}$ and at maximum width of the baffles the maximum outlet temperature of flowing air difference attained at mass flow rate $0.01 \mathrm{~kg} / \mathrm{s}$ and it is equal to $32.03 \mathrm{k}$ while the minimum increase in outlet temperature of air attained at mass flow rate $0.05 \mathrm{~kg} / \mathrm{s}$ and is equal to $6.97 \mathrm{k}$. At mass flow rate $0.03 \mathrm{~kg} / \mathrm{s}$ the effect of baffles width on the outlet temperature of the air has slight influence. The increase in baffles width more than $0.05 \mathrm{~m}$ on the outlet temperature of the flowing air has not noticeable. With the increase in solar intensity the outlet temperature of the flowing air increased due to more amount of solar energy absorption by solar air heater. At high mass flow rate with the increase in distance between the baffles the percentage in increase in temperature of the flowing air reduces sharply. By reducing the distance between the baffles or by attaching more number of baffles the out let temperature of the flowing air increases but at high mass flow rate the increase rate of outlet temperature of flowing air reduced. With the increase in number of fins the outlet temperature of the flowing air increases for both solar intensity radiations of $700 \mathrm{~W} / \mathrm{m}^{2}$ and $1000 \mathrm{~W} / \mathrm{m}^{2}$. It has been observed that beyond mass flow rate $0.03 \mathrm{~kg} / \mathrm{s}$ the increase in outlet temperature of flowing air with the increase in number of fins is not significant. The useful energy gains and efficiency increases with the attachment of fins and baffles. The pump work increases with the increase in mass flow rate, number of fins, width of baffles and by decreasing the distance between baffles. The difference between the useful energy gain and effective energy gain increases remarkably as well as the difference between the useful energy efficiency and effective energy efficiency increases remarkably. The increase in baffles width in comparison to the number of fins and distance between baffles results in higher increment of pressure drop especially in turbulent regime.

Bopche et al., [27] investigated experimentally heat transfer coefficient and friction factor of a solar absorber plate incorporated with inverted $u$ shaped turbulator. The experimentation has been carried out by varying the Reynolds number from 3800 to 18000 . The ratio of turbulator height to hydraulic mean diameter of the duct has been varied from 0.0186 to 0.03986 . The hydraulic diameter of the duct has been maintained constant throughout experimentation and it has been maintained 
at $37.63 \mathrm{~mm}$. The turbulator height has been varied from 0.7 to $1.5 \mathrm{~mm}$. The turbulator pitch to height ratio has been varied from 6.67 to 57.14. The pitch of the turbulator has been varied from 10 to $40 \mathrm{~mm}$. The angle of attack on turbulator $\alpha=90^{\circ}$ has been kept constant throughout the experimentation. The roughened wall has been supplied with uniform heat flux and the remaining three walls have been considered as insulated. From the investigation it has been concluded that the Nusselt number value for the relative roughness pitch and pitch 10,20,30 and 40mm increases with the increase in Reynolds number. The Nusselt number value is maximum when the pitch to height ratio is 6.67 and it starts to decrease with the increase in pitch to height ratio. The friction factor value for the turbulator roughened duct for wide range of pitch decreases with the increase in Reynolds number value due to decrease in thickness of viscous sub layer. The friction factor value decreases with the increase in relative roughness pitch value. The friction factor value is maximum at pitch to height ratio 6.67 and it is minimum at pitch to height ratio of 57.143 . The Nusselt number value increases with the increase in Reynolds number value. The Nusselt number value decreases with the increase in relative roughness pitch values. From the investigation it has been confirmed that for same pitch ratio $(P / e=20)$ at different pitch and turbulator height the performance of the duct has been significantly affected due to roughness height. From the observation it has been found out that the Nusselt number value for a fixed value of turbulator tip height to hydraulic mean diameter ratio at different value of pitch-height ratio increases with the increase in Reynolds number whereas the friction factor decreases. At Reynolds number value 8000 there was sudden drop in Nusselt number and friction factor value as the flow changes from transition to fully turbulent flow. The thermo hydraulic performance is maximum at pitch to height ratio 6.67. The heat transfer and friction factor of roughened duct from experimentation has been found to be 2.82 and 3.72 times higher as compared to the smooth duct.

Bekele et al., [55] investigated experimentally the effect of delta shaped obstacles mounted on the surface of the absorber plate of air heater. The investigation has been carried out by varying the Reynolds number from 3400-27600, relative longitudinal pitch of the obstacles from $3 / 2$ to $11 / 2$ and relative obstacles height from 0.25 to 0.75 . The investigation has been carried out for the air heater of aspect ratio 6:1. The studied has been carried out for a constant relative obstacles transverse pitch of $7 / 3$ and angle of attack of flow of an obstacles of $90^{\circ}$. The absorber plate has been supplied with constant heat flux of $800 \mathrm{~W} / \mathrm{m}^{2}$ throughout the experimentation. The heat transfer data of obstacles incorporated solar air heater has been compared with the smooth duct. It has been observed that the mechanism of flow separation, vortex formation and reattachment responsible for heat transfer enhancement in delta obstacles integrated solar air heater. From the investigation it has been concluded that with the increase in relative roughness height from 0.25 to 0.75 the Nusselt number as well as the pressure penalty increases for all relative longitudinal pitch and Reynolds number. The increase in relative roughness height increases the blockage to the flow hence the turbulence intensity in the flow increases. Hence the heat transfer coefficient increases in the solar air heater. The Nusselt number and friction factor increases when the relative longitudinal pitch decreases from $11 / 2$ to $3 / 2$. The decrease in relative longitudinal pitch increases the number of obstacles which further increases the reattachment point. Hence the friction factor and Nusselt number increases. The enhancement of heat transfer rate and friction factor is very high for relative longitudinal pitch of $3 / 2$ as compared to relative longitudinal pitch $7 / 2$ and $11 / 2$ The back flow in the upstream of obstacles increases after certain range of Reynolds number hence flow separation occurs. The flow separation pushes the fluid away from the heated surface near the obstacles and increases the reattachment length in the downstream of obstacles. Due the increase in reattachment length the effective heat transfer area of the heated absorber plate decreases. Hence the heat transfer enhancement rate and thermo hydraulic performance first increases with the increase in Reynolds 
number and then starts to fall. The heat transfer enhancement is maximum at relative longitudinal pitch of $3 / 2$ and relative roughness height of 0.75 at all value of Reynolds number. The overall maximum thermo hydraulic performance parameter is achieved at relative longitudinal pitch of $3 / 2$ and relative roughness height of 0.50 . The thermal performance of smooth duct is lower as compared to obstacles integrated air heater as the turbulence intensity in the flow of obstacles incorporated air heater is higher than smooth air heater.

Abdullah et al., [56] studied experimentally heat transfer efficiency of single pass solar air heater incorporated with turbulator and external mirror. The aluminum cans stacked over the surface of the absorber plate have been treated as turbulator. The single pass solar air heater has been incorporated with external bottom and top mirror to supply maximum solar irradiation to the absorber plate. The investigation has been carried out for three different arrangements. The first one is flat plate without can, second one is flat plate with inline cans arrangement and the third one is flat plate with staggered cans arrangement. The studied has been carried out by varying the mass flow rate from $0.02-0.05 \mathrm{~kg} / \mathrm{s}$ for the arrangement. From the investigation it has been observed that the solar intensity increases in the morning to a maximum value at noon and then it starts to fall onwards. The maximum value of $1250 \mathrm{~W} / \mathrm{m}^{2}$ has been achieved at noon. The flowing air has been also attained a maximum hourly temperature value at noon. The temperature difference for all the arrangements increases with time up to noon and starts to drop onwards. The temperature difference increases with the decrease in mass flow rate for all the arrangement as the residence time of cold fluid over the heat transferring surface increases. In staggered can arrange solar air heater as the air is properly distributed over the absorber plate the dead zone in the solar air heater reduces and turbulence increases.

Hence the temperature difference of staggered cans arranged solar air heater is better than inline can arranged solar air heater and flat plate solar air heater without can for a constant mass flow rate. The thermal efficiency of the single pass solar air heater for all arrangements increases when the mass flow rate increases from $0.02-0.05 \mathrm{~kg} / \mathrm{s}$. The thermal efficiency of staggered and inline can arranged single pass solar air heater without mirror has been enhanced by $10 \%$ and $7 \%$ over the flat single pass solar air heater at mass flow rate of $0.05 \mathrm{~kg} / \mathrm{s}$. The thermal efficiency of staggered can arranged single pass solar air heater with external top and bottom mirror increases by $32 \%$ then the flat plate single pass solar air heater at mass flow rate $0.05 \mathrm{~kg} / \mathrm{s}$. The thermal efficiency of inline can arranged single pass solar air heater with external top and bottom mirror increases by $28 \%$ over the flat plate solar air heater at mass flow rate $0.05 \mathrm{~kg} / \mathrm{s}$. The Nusselt number for all arrangement increases with the increase in mass flow rate. Whereas due to higher turbulence intensity the average Nusselt number of staggered can arranged solar air heater is higher as compared to the inline can arranged solar air heater and flat plate solar air heater.

Khawahaj et al., [57] investigated the thermal performance of a double flow solar air heater incorporated with 2, 4 and 6 number of fins and wire mesh has been inserted as absorber plate. From the investigation it has been concluded that the thermal efficiency increases with the mass flow rate (mass flow rate varies between $0.0121 \mathrm{~kg} / \mathrm{s}$ to $0.042 \mathrm{~kg} / \mathrm{s}$ ) for 2,4 and 6 fin type's solar heater. The maximum efficiencies of the solar air heater at mass flow rate $0.042 \mathrm{~kg} / \mathrm{s}$ for 2,4 and 6 fins are $75 \%$, $82.1 \%$ and $85.9 \%$ respectively. The collector efficiency increases with the increase in velocity of air. With the increase in number of fins the cross sectional area between the fin decreases and the velocity increases. The path length of the 6 fins solar air heater is more as compared to the 2 and 4 fins solar air heater hence more amount of heat will be extracted from the bed. The $\Delta T$ value decreases with the increase in mass flow rate. The maximum value of $\Delta T$ has been obtained between $12.00 \mathrm{~h}$ and $13.00 \mathrm{~h}$ of the local time as the intensity of solar radiation is maximum at that time. The introduction of wire mesh as absorber plate increases the heat transfer area hence the heat transfer 
between the air and bed increases. The maximum temperature difference between the inlet and out let air temperature of the 2.4 and 6 fins solar air heater was $53.3^{\circ} \mathrm{c}, 52.9^{\circ} \mathrm{c}$ and $62.1^{\circ} \mathrm{c}$ respectively.

Singh et al., [58] investigated the performance of curved solar air heater numerically. The solar air heater which has been taken for numerical investigation is same with the solar air heater which has been taken for experimental investigation by Mahboub et al., [59]. The investigation has been carried for different solar air heater named as: Flat plate smooth single pass (FPSP), flat plate smooth double pass (FPDP), curved plate smooth single pass (CPSP), curved plate v-corrugated single pass solar air heater. The relative height and relative pitch ratio of the absorber plate corrugation varies as 0.125-0.300 and 0.834-3.00 respectively. The numerical analysis has been taken for Reynolds number 2209, 3722 and 6058 corresponding to the mass flow rate of $0.0172,0.029$ and $0.0472 \mathrm{~kg} / \mathrm{sm} 2$ respectively. The unstructured meshing has been created on the two dimensional computational flow domain. The fine unstructured meshing near the sharp edged of the corrugated plate wall helps to predict the behavior of boundary layer and secondary vortices formation near to the wall of the absorber plate. The top wall of the absorber plate has been supplied with constant heat flux of 800,900 and $1000 \mathrm{~W} / \mathrm{m}^{2}$. The bottom and side wall of the duct has been considered smooth as well as adiabatic. The no slip boundary condition has been employed to bottom and side wall. The inlet and outlet of the flow domain has set with mass flow rate inlet and pressure outlet boundary condition respectively. The $\mathrm{k}-\epsilon$ turbulence model has been used to capture the turbulence flow structure. The governing equations which are used for flow simulation are continuity, momentum and energy equation. The velocity-pressure field coupling has been done by using SIMPLE algorithm. The convergence criteria for energy equation are $10^{-3}$ and $10^{-5}$ order for velocity component and momentum equation. When the fluid flows through the curved passage of solar air heater the centripetal and centrifugal force both plays an important role in enhancement of performance. When the mass flow rate is lower the intensity of centrifugal forces are less hence it does not affect the main velocity profile and the formation of secondary vortices are also small near the absorber plate. The curved plate double pass solar air heater has been found to be more effective than the curved plate single pass and flat double pass solar air heater in respect of air outlet temperature rise and thermal efficiency. The enhancement of thermal performance is due to more interaction time of air with hot absorber plate wall. The pressure drop in CPSP solar air heater is $25 \%$ higher than flat plate smooth single pass solar air heater and it is because of presence of adverse pressure gradient region near the absorber surface which gets compensated by generation of dean vortices. The curved plate $v$-corrugated smooth single pass solar air heater the flow reattachments on the absorber surface are strong enough to disturb the laminar sub layer region which increases the intensity of turbulence in the flow near the absorber wall. In case of curved plate semicircular smooth single pass solar air heater the reattachment point is present like curved plate v-corrugated smooth single pass solar air heater. The intensity of turbulence is lower in semicircular type solar air heater hence the heat transfer rate is less as compared to v-corrugated type solar air heater. Among all the solar air heater the curved plate v-corrugated smooth single pass solar air heater has maximum increase in thermal efficiency of $11.83 \%$ and temperature rise of $32.05 \%$ when the relative height and pitch ratios of 0.3 and 0.834 respectively. The maximum efficiency and rise in temperature in Curves plate v-corrugated smooth single SAH is due to (i) Availability of more surface area for heat transfer. (ii) degree of mixing is higher due to the presence of sharp edges as result of which huge quantity of heat transfer to the flowing air. (iv) The frequencies of exposure of the fresh air to hot absorber plate enhance the temperature at the outlet section. The value of surface average Nusselt number is maximum for curved plate smooth single pass solar air heater is due to continuous interaction of air stream flowing over the hot absorber plate. The surface average Nusselt number of curved plate double pass smooth solar air heater is slightly less because (i) At the first phase due to 
higher temperature gradient between the air and the heated surface higher heat transfer occurs (ii) At the second pass as the same air flowing over the lower surface of the heated absorber plate hence temperature gradient is less. In case of semicircular and v-corrugated smooth curved single pass solar air heater the air will be in stagnant condition as they will be trapped in between the grooves of the corrugated solar heater for which the Nusselt number value decreases. The high turbulence occurs at the sharp tip of the grooves which enhance the mixing rate in the flow as result of which the air temperature increases. The minimum value of relative pitch ratio produces higher pressure drop. More the obstruction more will be the pressure drop. The curved plate v-corrugated solar smooth single pass solar air heater has higher pressure drop rate due to the presence of more height grooves. The increase in relative height ratio and relative pitch ratio increases the surface area of absorber plate which is exposed to the air. It increases the turbulence region near the wall of the absorber plate. The turbulence increases the mixing rate of fluid particle. The fresher air temperature increase due to flow of cold fluid over hot surface as heat extraction rate from hot surface increases. Hence the thermal efficiency increases. The turbulence rate due to sharp edges of v-grooves with more height with fine pitches increases the curves plate $v$-corrugated smooth single pass solar air heater thermal efficiency increases. The total pressure coefficient is high in case of curved plate v-corrugated smooth single pass solar air heater due to the formation of more number of vortices in the flow near to the absorber plate wall and obstruction offered by corrugation. The turbulent kinetic energy is maximum at relative pitch ratio of 0.834 and next maximum at relative pitch ratio of 1.25 for curved v-corrugated smooth single pass solar air heater when the mass flow rate is $0.0472 \mathrm{~kg} / \mathrm{sm}^{2}$. The turbulent kinetic energy is maximum near to tip of the $\mathrm{v}$ - grooves of the hot corrugated convex shape hot absorber plate.

Priyam et al., [28] investigated analytically the performance of finned absorber solar air heater. The effects of mass flow rate, fin spacing on thermal performance of solar air heater has been studied and compared it with the plain solar air heater. The solar absorber plate has been incorporated with two transversely positioned wavy fins. The 5 different fins spacing has been taken for investigation. The top surface of the absorber plate has been supplied with uniform heat flux. The bottom side of the absorber plate is insulated. The total loss coefficient for a wavy finned solar air heater decreases with the increase in mass flow rate for all value of fin spacing. The total loss coefficient is minimum for fin spacing $5 \mathrm{~cm}$ and it is about $5.9 \mathrm{~W} / \mathrm{m}^{2} \mathrm{k}$. The collector efficiency factor for a wavy finned solar air heater increases significantly for the entire range of mass flow rate and for all value of fin spacing. The decrease in fin spacing increases the effective heat transfer area hence the maximum enhancement of collector efficiency factor has been found 1.84 times with the fin spacing of $1 \mathrm{~cm}$ as compared to the plane solar air heater. The collector heat removal factor for plane as well as finned incorporated solar air heater increases gradually with the increase in mass flow rate for all fin spacing. The maximum heat removal rate of the collector is about 2.03 times than the plain solar air heater when the fin spacing and mass flow rate is $1 \mathrm{~cm}$ and $0.0834 \mathrm{~kg} / \mathrm{s}$ respectively. The thermal efficiency when the fin spacing is $1 \mathrm{~cm}$ is highest for all range of mass flow rates due to high heat transfer area. The thermal efficiency is about $35.88 \%$ as compared to plane solar air heater when the mass flow rate is $0.0138 \mathrm{~kg} / \mathrm{s}$ and fin spacing is $1 \mathrm{~cm}$. It is $20.17 \%$ when the fin spacing is $5 \mathrm{~cm}$ and mass flow rate is $0.0138 \mathrm{~kg} / \mathrm{s}$. The pressure drop is maximum when the fin spacing is minimum hence for fin spacing $1 \mathrm{~cm}$ the pressure drop is maximum. The rise in temperature decreases with the increase in mass flow rate for various fin spacing. The maximum rise in temperature is obtained at fin spacing $1 \mathrm{~cm}$ due to maximum energy gain by the absorber. The effective fin efficiency increases with the increase in mass flow rate attains a maximum and then starts decreasing on further increase of mass flow rate. The maximum effective efficiency is obtained when fin spacing is $1 \mathrm{~cm}$. The effective efficiency is highest at fin spacing $1 \mathrm{~cm}$ due to increase in heat transfer area. The effective efficiency is $35.83 \%$ then the 
plain solar air heater when the mass flow rate is $0.0138 \mathrm{~kg} / \mathrm{s}$ and fin spacing is $1 \mathrm{~cm}$. The effective efficiency is about $17.56 \%$ then the plain solar air heater when the mass flow rate is $0.0834 \mathrm{~kg} / \mathrm{s}$ and fin spacing is $1 \mathrm{~cm}$. The effect of heat transfer coefficient dominates the friction loss up to maxima as a result of which the pressure drop will be minimum. The friction factor dominates the heat transfer coefficient after maxima due to which the maximum pressure drop occurs and effective efficiency decreases. The thermal and thermo hydraulic performance increases with the increase in mass flow rate when the fin spacing is $1 \mathrm{~cm}$. There is a separation between the thermal and thermo hydraulic efficiencies after certain mass flow rate as some energy has been spent to overcome the friction. The maximum thermal and thermo hydraulic efficiency is about 2.98 times and 2.8 times that of the plane solar air heater.

\subsection{Packed Bed Solar Air Heater}

Dhiman et al., [60] investigated the thermal performance of parallel flow packed bed solar air heater. The parallel flow packed bed solar air heater upper channel has been packed with porous material. The thermal performance of the double flow porous material filled solar air heater has been compared with the non-porous material double flow system solar air heater. An analytical model capable of describing temperature distribution and heat transfer characteristics of parallel flow packed bed solar air heater has been taken to study the effect of porosity and mass flow rate on thermal performance. The governing equation involved the heat and mass exchanged has been solved using an iterative method. The heat removal rate directly depends on the mass flow rate hence the thermal efficiency increases with the increase in mass flow rate. The thermal efficiency of packed bed double flow solar air heater is higher as compared to the double flow solar air heater without porous material. Due to the presence of porous material the heat transfer area increases. So depending on the porosity level the thermal efficiency of the double flow packed bed solar heater is 3-10\% higher as compared to the solar air heater without porous media. The thermal power output increment rate of the solar air heater with packed bed with the increase in mass flow rate is higher as compared to the double flow solar air heater without porous media. The outlet temperature of the air stream flowing in the upper channel of the packed bed double flow solar air heater is higher as compared to the outlet temperature of the air flowing in the lower channel. This is because of higher volumetric heat transfer coefficient in the upper channel due to the presence of porous material. The outlet temperature of the air flowing in the upper channel of the double flow solar air heater without porous media is less than the air flowing in the lower channel. The outlet temperature of air flowing in the lower channel is higher as the heat loss is less due to the presence of insulation. The effective efficiency of the packed bed double flow solar air heater increases with the increase in mass flow rate when the porosity level is higher than $80 \%$. The effective efficiency of packed bed double flow solar air heater decreases with the increase in mass flow rate when the porosity level is less than $80 \%$.

\subsection{Solar Air Heater with Thermal Storage}

Khadraoui et al., [61] investigated experimentally the effect of use of phase change material on the efficiency of the simple solar air heater. The solar air heater is filled with paraffin wax which has been used as latent heat storage. The efficiency of both solar air heaters with phase change material and without phase change material has been studied and compared. For evaluating the efficiency two session has been taken one is from morning 6 ' 0 ' clock to evening 6 ' 0 ' clock (charging) and another one is evening 6 ' $o$ ' clock to next day morning 6 ' 0 ' clock (discharging). During charging the 
inlet and out of solar air heater with PCM has been closed. During discharging the air will be passed over the black absorber surface of the PCM cavity. The air which is passed over it extract heat. The efficiency of solar air heater with PCM cavity has been investigated for mass flow rate of $0 \mathrm{~kg} / \mathrm{s}, 0.018 \mathrm{~kg} / \mathrm{s}$ and $0.055 \mathrm{~kg} / \mathrm{s}$. The out let temperature of solar air heater with PCM cavity increases up to certain mass flow rate after that it starts to fall. The down fall of out let temperature of the solar air heater with PCM cavity in the evening at mass flow rate $0.018 \mathrm{~kg} / \mathrm{s}$ is less. When the air mass flow rate is $0 \mathrm{~kg} / \mathrm{s}$ that means natural convection the air flow over it is not sufficient to extract heat from the solar air heater with PCM cavity. When the mass flow rate is $0.055 \mathrm{~kg} / \mathrm{s}$ the out let temperature drop sharply as the residence time of air over the heated black surface absorber with PCM cavity decreases or due to response time between the heated surface and air is less. The global radiation intensity and the ambient temperature at mid-day reach to a maximum value of $790 \mathrm{~W} / \mathrm{m}^{2}$ and $35^{\circ} \mathrm{C}$ respectively. The temperature of solar air heater with $\mathrm{PCM}$ cavity reaches to a maximum value of $65^{\circ} \mathrm{C}$ at $13.00 \mathrm{hrs}$ during charging. The outlet temperature of solar air heater with PCM during discharge period is $7-10^{\circ} \mathrm{C}$ higher than the ambient air temperature. Both the solar air heater with PCM cavity and without cavity increases with the increase in intensity of solar radiation. The temperature of solar air heater without $\mathrm{PCM}$ reaches to a value of $71^{\circ} \mathrm{C}$ and with $\mathrm{PCM}$ reaches to a value of $65^{\circ} \mathrm{C}$ respectively during charging process. During discharging process the out let temperature of the solar air heater with PCM cavity is higher than that of solar air heater with PCM and it is about $3-7^{\circ} \mathrm{C}$ higher. The outlet temperature of solar air heater with PCM maintained a constant value of $34^{\circ} \mathrm{C}$. It is because of thermal energy stored in the paraffin wax. The outlet temperature of solar air heater without PCM decreases progressively after 2 hours from the sun set. The daily energy efficiency of the solar air heater with PCM is about $34 \%$ whereas the daily efficiency of the solar air heater without PCM is $17 \%$. The difference in daily efficiency of solar air heater is due to two reasons: (i) The paraffin wax can store more amount of latent heat. (ii) The air thermal capacity is lower and there is lower heat transfer coefficient in between the air and the absorber plate. The solar air heater with PCM creates passive dehumidification process at night due to increase of the outlet temperature. For drying purpose, the low relative humidity is the favorable condition as it increases the evaporation rate.

Krishnananth et al., [62] investigated the thermal performance of a double pass solar air heater integrated with a thermal storage system. The paraffin wax filled aluminum capsule has been taken as thermal storage system. The three different configurations have been taken for investigation named as configuration 1 , configuration 2 and configuration 3 . In configuration 1 and 2 the aluminum capsule filled with paraffin wax has been placed over and below the absorber plate respectively. Whereas in configuration 3 the aluminum capsule filled with paraffin wax has been placed over the back plate. From the investigation it has been concluded that the maximum solar radiation intensity is achieved at $12.30 \mathrm{pm}$ and it is $900 \mathrm{~W} / \mathrm{m}^{2}$. The ambient air temperature increases from $30^{\circ} \mathrm{C}$ to $40^{\circ} \mathrm{C}$ from morning to evening. The temperature of the absorber plate is highest in solar air heater and it reached at maximum value of $70^{\circ} \mathrm{C}$ at noon. The temperature of the exit air is varied with the surface temperature of the capsule and it reached at maximum value of $55^{\circ} \mathrm{C}$ around noon. The rise in all temperature after 8A.M is in appreciable amount. The fall of all temperature after 5P.M reached at lower value. The maximum temperature of air achieved by solar air heater without storage system is less as compared to solar air heater with storage system. The fall of temperature in the evening is higher for solar heater without storage system. The performance of configuration 2 is better and the air is maintained at maximum value from 9A.M to 7P.M. The efficiency of solar air heater without storage system is proportional to the solar radiation received. Whereas solar air heater with storage system efficiency is higher and the efficiency increased during the evening hours as the excess energy stored in the morning is released during evening. 


\section{Future Scope}

Based on the literature survey following suggestions and recommendations have been made for the improvement in solar air heater

i. More research may be carried out for the solar air heater with a reflector as more amount of radiation can be absorbed by the absorber plate as well as transfer to the flowing air as compared to the fixed solar air heater.

ii. More studied must be carried out on material and its geometry to improve the thermal performance of solar air heaters.

iii. The study must be carried out to obtain new geometry that is easy to fabricate and simple in configuration.

iv. More investigation for making different configurations of double-pass solar air heaters roughened with artificial elements also is a research area to enhance the thermal performance.

v. Different types of packing material in the case of double pass packed bed solar air heater must be investigated.

vi. Nowadays, nanofluid is playing an essential role in enhancing heat transfer. Therefore, more studies may be required on nanofluid-coated absorber plate solar air heater to improve the thermal performance of solar air heater, as the coating of nanofluid over the absorber plate enhance the heat transfer area.

vii. Comprehensive studies on different roughness geometries such as a rib, corrugated, arc, finned and v-shaped groove have been investigated. More studies must be focused on dimple incorporated absorber plate solar air heaters. The dimple has higher thermal performance as compared to other roughness geometry as well, as the rate of increase in friction factor concerning the rate of increase in heat transfer rate is less as compared to other roughness.

viii. The development of dimple and protrusion is easier as well as it does not add extra weight to the system and make the system light weight. It is essential to implement different dimple geometry on absorber plate in solar air heater for enhancement of thermal and thermo hydraulic efficiency.

\section{Conclusions}

In this paper an attempt has been made to report the heat transfer and friction characteristics of various artificial roughness elements such as rib, turbulator, fins, wire matrix, dimple, protrusion, arc etc. Following conclusions could be drawn from the present study

i. The addition of artificial roughness increases absorber duct outlet temperature; simultaneously it increases the pressure drop. Hence the effectiveness of the solar air heater is decided by the thermo hydraulic enhancement factor.

ii. It is generally noticed that with the increase in Reynolds number value the thermo hydraulic performance decreases due the increase in pressure penalty. Hence an efficient design that enhancedthe thermo hydraulic performance with the increase in Reynolds number value is required.

iii. The shape, size and orientation of the artificial roughness plays critical role in the effectiveness of the heat transfer hence it is a good technique to improve thermal performance of solar air heater by optimize the geometries. 
iv. From the data reported by various investigators it has been observed that the rise in Nusselt number value for dimple is higher as compared to other artificial roughness geometry.

v. The rate of increase in friction factor value with the increase in Reynolds number as compared to rate of increase in Nusselt number is small in case of dimple roughness.

Different configurations and arrangements of dimple imprinted solar absorber plate can be used for future studies to enhance the thermal performance in solar air heaters. Similarly, coated absorber plate can be used in solar air heaters to analyze the thermal efficiency.

\section{Acknowledgement}

This research was funded by a grant from Ministry of Higher Education of Malaysia (FRGS Grant R.J130000.7824.4X172).

\section{References}

[1] Muhieldeen, Mohammed W., Lim Chong Lye, M. S. S. Kassim, Tey Wah Yen, and K. H. Teng. "Effect of Rockwool Insulation on Room Temperature Distribution." Journal of Advanced Research in Experimental Fluid Mechanics and Heat Transfer 3, no. 1 (2021): 9-15.

[2] Saini, R. P., and S. K. Singal. "A review on roughness geometry used in solar air heaters." Solar Energy 81, no. 11 (2007): 1340-1350. https://doi.org/10.1016/i.solener.2007.01.017

[3] Lubis, Hamzah. "Renewable Energy of Rice Husk for Reducing Fossil Energy in Indonesia." Journal of Advanced Research in Applied Sciences and Engineering Technology 11, no. 1 (2018): 17-22.

[4] Yacob, Noraishah Shafiqah, and Hassan Mohamed. "Investigation of Palm Oil Wastes Characteristics for Co-Firing with Coal." Journal of Advanced Research in Applied Sciences and Engineering Technology 23, no. 1 (2021): 34-42. https://doi.org/10.37934/araset.23.1.3442

[5] Khattak, Muhammad Adil, Mohammad Azfar Haziq Ayoub, Muhammad Ariff Fadhlillah Abdul Manaf, Mohd Faidhi Mahru, Mohd Ridwan Mohd Juhari, Mira Idora Mustaffa, and Suhail Kazi. "Global energy security and European Union: A review." Journal of Advanced Research in Applied Sciences and Engineering Technology 11, no. 1 (2018): 64-81.

[6] Hayder, G., and P. Puniyarasen. "Identification and evaluation of wastes from biodiesel production process." Journal of Advanced Research in Applied Sciences and Engineering Technology 3, no. 1 (2016): 21-29.

[7] Abhishek, Saxena, P. Varun, and A. A. El-Sebaii. "A thermodynamic review of solar air heaters." Renewable and Sustainable Energy Reviews 43 (2015): 863-890. https://doi.org/10.1016/i.rser.2014.11.059

[8] Ghritlahre, Harish Kumar, and Piyush Kumar Sahu. "A comprehensive review on energy and exergy analysis of solar air heaters." Archives of Thermodynamics (2020): 183-222.

[9] Abd El-Hameed, Afaf M., and Y. A. Abdel-Aziz. "Aluminium Alloys in Space Applications: A Short Report." Journal of Advanced Research in Applied Sciences and Engineering Technology 22, no. 1 (2021): 1-7.

[10] Ghritlahre, Harish K., and Radha K. Prasad. "Investigation on heat transfer characteristics of roughened solar air heater using ANN technique." International Journal of Heat and Technology 36, no. 1 (2018): 102-110. https://doi.org/10.18280/ijht.360114

[11] Ghritlahre, Harish Kumar, and Radha Krishna Prasad. "Investigation of thermal performance of unidirectional flow porous bed solar air heater using MLP, GRNN, and RBF models of ANN technique." Thermal Science and Engineering Progress 6 (2018): 226-235. https://doi.org/10.1016/j.tsep.2018.04.006

[12] Ghritlahre, Harish Kumar, and Radha Krishna Prasad. "Modelling of back propagation neural network to predict the thermal performance of porous bed solar air heater." Archives of Thermodynamics 40, no. 4 (2019).

[13] Bhushan, Brij, and Ranjit Singh. "A review on methodology of artificial roughness used in duct of solar air heaters." Energy 35, no. 1 (2010): 202-212. https://doi.org/10.1016/i.energy.2009.09.010

[14] Oo, Ye Min, Makatar Wae-hayee, and Chayut Nuntadusit. "Experimental and Numerical Study on the Effect of Teardrop Dimple/Protrusion Spacing on Flow Structure and Heat Transfer Characteristics." Journal of Advanced Research in Experimental Fluid Mechanics and Heat Transfer 2, no. 1 (2020): 17-32.

[15] Ghritlahre, Harish Kumar. "Heat transfer and friction factor characteristics investigation of roughened solar air heater using arc shaped wire rib roughness." International Journal of Ambient Energy just-accepted (2021): 1-36. https://doi.org/10.1080/01430750.2021.1934115

[16] Tanda, Giovanni. "Performance of solar air heater ducts with different types of ribs on the absorber plate." Energy 36, no. 11 (2011): 6651-6660. https://doi.org/10.1016/i.energy.2011.08.043 
[17] Layek, A., J. S. Saini, and S. C. Solanki. "Heat transfer coefficient and friction characteristics of rectangular solar air heater duct using rib-grooved artificial roughness." Int J Heat Mass Transf 50 (2007): 4845-4854. https://doi.org/10.1016/j.solener.2005.08.006

[18] Gawande, Vipin B., A. S. Dhoble, D. B. Zodpe, and Sunil Chamoli. "Experimental and CFD investigation of convection heat transfer in solar air heater with reverse L-shaped ribs." Solar Energy 131 (2016): $275-295$. https://doi.org/10.1016/i.solener.2016.02.040

[19] Layek, Apurba, J. S. Saini, and S. C. Solanki. "Effect of chamfering on heat transfer and friction characteristics of solar air heater having absorber plate roughened with compound turbulators." Renewable Energy 34, no. 5 (2009): 1292-1298. https://doi.org/10.1016/S0360-5442(00)00062-1

[20] Momin, Abdul-Malik Ebrahim, J. S. Saini, and S. C. Solanki. "Heat transfer and friction in solar air heater duct with V-shaped rib roughness on absorber plate." International journal of heat and mass transfer 45, no. 16 (2002): 3383 3396. https://doi.org/10.1016/S0017-9310(02)00046-7

[21] Aharwal, K. R., Bhupendra K. Gandhi, and J. S. Saini. "Heat transfer and friction characteristics of solar air heater ducts having integral inclined discrete ribs on absorber plate." International Journal of Heat and Mass Transfer 52, no. 25-26 (2009): 5970-5977. https://doi.org/10.1016/j.ijheatmasstransfer.2009.05.032

[22] Singh, Satyender. "Experimental and numerical investigations of a single and double pass porous serpentine wavy wiremesh packed bed solar air heater." Renewable Energy 145 (2020): 1361-1387. https://doi.org/10.1016/j.renene.2019.06.137

[23] Saini, R. P., and Jitendra Verma. "Heat transfer and friction factor correlations for a duct having dimple-shape artificial roughness for solar air heaters." Energy 33, no. $8 \quad$ (2008): $1277-1287$. https://doi.org/10.1016/i.energy.2008.02.017

[24] Lanjewar, Atul, J. L. Bhagoria, and R. M. Sarviya. "Experimental study of augmented heat transfer and friction in solar air heater with different orientations of W-Rib roughness." Experimental Thermal and Fluid Science 35, no. 6 (2011): 986-995. https://doi.org/10.1016/i.expthermflusci.2011.01.019

[25] Karim, Md Azharul, and M. N. A. Hawlader. "Performance investigation of flat plate, v-corrugated and finned air collectors." Energy 31, no. 4 (2006): 452-470. https://doi.org/10.1016/i.energy.2005.03.007

[26] Choudhury, C., and H. P. Garg. "Design analysis of corrugated and flat plate solar air heaters." Renewable energy 1 , no. 5-6 (1991): 595-607. https://doi.org/10.1016/0960-1481(91)90003-8

[27] Bopche, Santosh B., and Madhukar S. Tandale. "Experimental investigations on heat transfer and frictional characteristics of a turbulator roughened solar air heater duct." International Journal of Heat and Mass Transfer 52, no. 11-12 (2009): 2834-2848. https://doi.org/10.1016/j.ijheatmasstransfer.2008.09.039

[28] Priyam, Abhishek, and Prabha Chand. "Thermal and thermohydraulic performance of wavy finned absorber solar air heater." Solar Energy 130 (2016): 250-259. https://doi.org/10.1016/j.solener.2016.02.030

[29] Alam, Tabish, and Man-Hoe Kim. "Heat transfer enhancement in solar air heater duct with conical protrusion $\begin{array}{lllll}\text { roughness } \quad \text { ribs." Applied } \quad \text { Thermal } & \text { (2017): }\end{array}$ https://doi.org/10.1016/j.applthermaleng.2017.07.181

[30] Sahu, M. M., and J. L. Bhagoria. "Augmentation of heat transfer coefficient by using 90 broken transverse ribs on absorber plate of solar air heater." Renewable energy 30, no. 13 (2005): 2057-2073. https://doi.org/10.1016/j.renene.2004.10.016

[31] Kumar, Anil, R. P. Saini, and J. S. Saini. "Heat and fluid flow characteristics of roughened solar air heater ducts-A review." Renewable Energy 47 (2012): 77-94. https://doi.org/10.1016/j.renene.2012.04.001

[32] Ghritlahre, Harish Kumar, Piyush Kumar Sahu, and Subhash Chand. "Thermal performance and heat transfer analysis of arc shaped roughened solar air heater-An experimental study." Solar Energy 199 (2020): $173-182$. https://doi.org/10.1016/i.solener.2020.01.068

[33] Kumar, Anil. "Analysis of heat transfer and fluid flow in different shaped roughness elements on the absorber plate solar air heater duct." Energy Procedia 57 (2014): 2102-2111. https://doi.org/10.1016/i.egypro.2014.10.176

[34] Prasad, Radha K., and Mukesh K. Sahu. "Entropy generation and thermodynamic analysis of solar air heaters with artificial roughness on absorber plate." Archives of thermodynamics 38, no. 3 (2017). https://doi.org/10.1515/aoter-2017-0014

[35] Promvonge, P., C. Khanoknaiyakarn, S. Kwankaomeng, and C. Thianpong. "Thermal behavior in solar air heater channel fitted with combined rib and delta-winglet." International Communications in Heat and Mass Transfer 38, no. 6 (2011): 749-756. https://doi.org/10.1016/j.icheatmasstransfer.2011.03.014

[36] Skullong, Sompol, Sutapat Kwankaomeng, Chinaruk Thianpong, and Pongjet Promvonge. "Thermal performance of turbulent flow in a solar air heater channel with rib-groove turbulators." International Communications in Heat and Mass Transfer 50 (2014): 34-43. https://doi.org/10.1016/i.icheatmasstransfer.2013.11.001

[37] Sethi, Muneesh, and N. S. Thakur. "Correlations for solar air heater duct with dimpled shape roughness elements on absorber plate." Solar Energy 86, no. 9 (2012): 2852-2861. https://doi.org/10.1016/i.solener.2012.06.024 
[38] Yadav, Sanjay, and Maneesh Kaushal. "Nusselt number and friction factor correlations for solar air heater duct having protrusions as roughness elements on absorber plate." Experimental Thermal and Fluid Science 44 (2013): 34-41. https://doi.org/10.1016/j.expthermflusci.2012.05.011

[39] Bhushan, Brij, and Ranjit Singh. "Thermal and thermohydraulic performance of roughened solar air heater having protruded absorber plate." Solar energy 86, no. $11 \quad$ (2012): 3388-3396. https://doi.org/10.1016/i.solener.2012.09.004

[40] Pakdaman, M. Fakoor, A. Lashkari, H. Basirat Tabrizi, and R. Hosseini. "Performance evaluation of a naturalconvection solar air-heater with a rectangular-finned absorber plate." Energy conversion and management 52, no. 2 (2011): 1215-1225. https://doi.org/10.1016/i.enconman.2010.09.017

[41] Sawhney, J. S., Rajesh Maithani, and Sunil Chamoli. "Experimental investigation of heat transfer and friction factor characteristics of solar air heater using wavy delta winglets." Applied thermal engineering 117 (2017): $740-751$. https://doi.org/10.1016/i.applthermaleng.2017.01.113

[42] Karim, M. A., and MiNAr Hawlader. "Development of solar air collectors for drying applications." Energy conversion and management 45, no. 3 (2004): 329-344. https://doi.org/10.1016/S0196-8904(03)00158-4

[43] Kabeel, Abd Elnaby, Mofreh H. Hamed, Z. M. Omara, and A. W. Kandeal. "Solar air heaters: Design configurations, improvement methods and applications-A detailed review." Renewable and Sustainable Energy Reviews 70 (2017): 1189-1206. https://doi.org/10.1016/i.rser.2016.12.021

[44] Kumar, Dhananjay, and Laljee Prasad. "Heat Transfer Augmentation of Various Roughness Geometry Used in Solar Air Heaters." Int. J. Mech. Eng. Technol. 12 (2017): 491-508.

[45] Kabeel, A. E., and K. Mečárik. "Shape optimization for absorber plates of solar air collectors." Renewable Energy 13, no. 1 (1998): 121-131. https://doi.org/10.1016/S0960-1481(97)00034-7

[46] Hachemi, A. "Thermal performance enhancement of solar air heaters, by a fan-blown absorber plate with rectangular fins." International journal of energy research 19, no. 7 (1995): 567-577. https://doi.org/10.1002/er.4440190703

[47] Kabeel, A. E., A. Khalil, S. M. Shalaby, and M. E. Zayed. "Investigation of the thermal performances of flat, finned, and v-corrugated plate solar air heaters." Journal of Solar Energy Engineering 138, no. 5 (2016): 051004. https://doi.org/10.1115/1.4034027

[48] Akpinar, Ebru Kavak, and Fatih Koçyiğit. "Experimental investigation of thermal performance of solar air heater having different obstacles on absorber plates." International Communications in Heat and Mass Transfer 37, no. 4 (2010): 416-421. https://doi.org/10.1016/i.icheatmasstransfer.2009.11.007

[49] Ong, K. S. "Thermal performance of solar air heaters-Experimental correlation." Solar Energy 55, no. 3 (1995): 209-220. https://doi.org/10.1016/0038-092X(95)00027-0

[50] Yeh, Ho-Ming, and Tong-Tshien Lin. "Efficiency improvement of flat-plate solar air heaters." Energy 21, no. 6 (1996): 435-443. https://doi.org/10.1016/0360-5442(96)00008-4

[51] Chabane, Foued, Noureddine Moummi, and Said Benramache. "Experimental study of heat transfer and thermal performance with longitudinal fins of solar air heater." Journal of advanced research 5, no. 2 (2014): $183-192$. https://doi.org/10.1016/i.jare.2013.03.001

[52] Behura, Arun Kumar, Sachindra Kumar Rout, Himanshu Pandya, and Ashiwini Kumar. "Thermal analysis of three sides artificially roughened solar air heaters." Energy Procedia 109 (2017): 279-285. https://doi.org/10.1016/i.egypro.2017.03.066

[53] Gao, Wenfeng, Wenxian Lin, and Enrong Lu. "Numerical study on natural convection inside the channel between the flat-plate cover and sine-wave absorber of a cross-corrugated solar air heater." Energy Conversion and Management 41, no. 2 (2000): 145-151. https://doi.org/10.1016/S0196-8904(99)00098-9

[54] Mohammadi, K., and M. Sabzpooshani. "Comprehensive performance evaluation and parametric studies of single pass solar air heater with fins and baffles attached over the absorber plate." Energy 57 (2013): $741-750$. https://doi.org/10.1016/i.energy.2013.05.016

[55] Bekele, Adisu, Manish Mishra, and Sushanta Dutta. "Effects of delta-shaped obstacles on the thermal performance of solar air heater." Advances in Mechanical Engineering 3 (2011): 103502. https://doi.org/10.1155/2011/103502

[56] Abdullah, A. S., M. I. Amro, M. M. Younes, Z. M. Omara, A. E. Kabeel, and F. A. Essa. "Experimental investigation of single pass solar air heater with reflectors and turbulators." Alexandria Engineering Journal 59, no. 2 (2020): 579587. https://doi.org/10.1016/j.aej.2020.02.004

[57] El-Khawajah, M. F., L. B. Y. Aldabbagh, and F. Egelioglu. "The effect of using transverse fins on a double pass flow solar air heater using wire mesh as an absorber." Solar energy 85, no. 7 (2011): 1479-1487. https://doi.org/10.1016/j.solener.2011.04.004

[58] Singh, Ajeet Pratap, and O. P. Singh. "Performance enhancement of a curved solar air heater using CFD." Solar Energy 174 (2018): 556-569. https://doi.org/10.1016/j.solener.2018.09.053 
[59] Mahboub, Chawki, Noureddine Moummi, Abdelhafid Brima, and Abdelhafid Moummi. "Experimental study of new solar air heater design." International journal of green energy 13, no. 5 (2016): 521-529. https://doi.org/10.1080/15435075.2014.968922

[60] Dhiman, Prashant, N. S. Thakur, Anoop Kumar, and Satyender Singh. "An analytical model to predict the thermal performance of a novel parallel flow packed bed solar air heater." Applied energy 88, no. 6 (2011): 2157-2167. https://doi.org/10.1016/i.apenergy.2010.12.033

[61] El Khadraoui, Aymen, Salwa Bouadila, Sami Kooli, Amenallah Guizani, and Abdelhamid Farhat. "Solar air heater with phase change material: An energy analysis and a comparative study." Applied Thermal Engineering 107 (2016): 1057-1064. https://doi.org/10.1016/i.applthermaleng.2016.07.004

[62] Krishnananth, S. S., and K. Kalidasa Murugavel. "Experimental study on double pass solar air heater with thermal energy storage." Journal of King Saud University-Engineering Sciences 25, no. 2 (2013): 135-140. https://doi.org/10.1016/i.jksues.2012.05.004 